\title{
Effect of Limbal Incision Positions on Corneal Q-Value in Cataract Surgery: A Retrospective Study of Monofocal IOL and Trifocal IOL
}

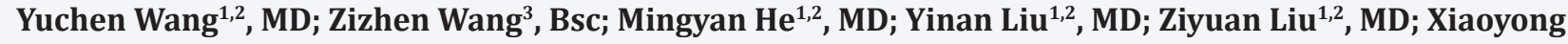 \\ Chen $^{1,2 *}$, MD, PhD; Wei Wang ${ }^{1,2}$, MD, PhD \\ ${ }^{1}$ Department of Ophthalmology, Peking University Third Hospital, 49 North Garden Road, Haidian District, Beijing 100191, P R China \\ ${ }^{2}$ Beijing key Laboratory of restoration of damaged ocular nerve, Peking University Third hospital, 49 North Garden Road, Haidian District, Beijing \\ 100191, P R China
}

${ }^{3}$ Peking University Health Science Center, 38 North Garden Road, Haidian District, Beijing 100191, P R China

Yuchen Wang and Zizhen Wang contributed equally to this work

\section{ABSTRACT}

Purpose: To observe the changes in corneal $Q$ value preoperatively and postoperatively in cataract patients and to further explore the influence of incision positions on $Q$ value.

Setting: The Peking University Third Hospital, Beijing, China.

Design: Retrospective nonrandomized study.

Methods: Eyes that implanted intraocular lens (IOL) ZCB00 or Zeiss 839 were included. The corneal $\mathrm{Q}$ values were compared preoperatively, 1 week and 1 month postoperatively, the incision positions were divided into groups to review the influence on $Q$ values.

Results: We finally analyzed 158 eyes of 103 patients, the corneal Q values decreased significantly postoperatively, and the $Q$ values tend to be stable at 1 month after surgery. Moreover, the $Q$ values in superior incision group and temporal incision group were lower than that in nasal, nasal and temporal arc group at both 1 week and 1 month after surgery with statically significance; the reduction of angle kappa postoperatively had obvious advantages in the superior and temporal incision group.

Conclusions: Corneal Q-value would change after cataract surgery, and the influence of incision position on corneal Q-value need still to be further clear, clarifying the influence of surgical operation, especially incision making, on corneal Q-value will achieve better vision and visual quality after cataract surgery.

KEYWORDS: Intraocular lens; Corneal Q value; Incision position; High order aberration; Visual quality

ABBREVIATIONS: IOL: Intraocular Lens

\section{INTRODUCTION}

In recent years, as people's living standards have improved, people's pursuit of visual quality has increased year by year. Cataract surgery
Vol No: 06, Issue: 01

Received Date: July 16, 2021

Published Date: August 16, 2021

\section{*Corresponding Author}

Xiaoyong Chen

Department of Ophthalmology, Peking University Third Hospital, Beijing key laboratory of restoration of damaged ocular nerve, Peking University Third hospital, 49 North Garden Road, Haidian District, Beijing 100191, P R China, Tel: +86-15611908041

E-mail: drchxy@126.com

Citation: Chen X, et al. (2021). Effect of limbal incision positions on corneal Q-value in cataract surgery: a retrospective study of monofocal IOL and trifocal IOL. Mathews J Ophthalmol. (6)1:26.

Copyright: Chen X, et al. (C) (2021). This is an open-access article distributed under the terms of the Creative Commons Attribution License, which permits unrestricted use, distribution, and reproduction in any medium, provided the original author and source are credited. 
has developed from vision restoration surgery to refractive cataract surgery today. Factors affecting postoperative visual quality, such as postoperative visual acuity, aberration and contrast sensitivity, have been increasingly emphasized. However, while realizing refractive cataract surgery, the cornea, as the main component of the human eye's refractive system, should be taken into consideration in the design of the intraocular lens (IOL). As well as the diopters and the operation method need be considered to realize the mutual matching of the cornea and the IOL.

Since the human cornea is not a perfect spherical surface, the anterior surface of the cornea gradually flattens from the center to the periphery to form a slightly elliptical aspherical surface, which is coordinated with other refractive components (such as lens, aqueous humor and vitreous body) to ensure the quality of retinal imaging $[1,2]$. The corneal $Q$ value is a parameter used to describe the aspheric morphology of the anterior surface of the cornea, which expresses the degree of corneal curvature inconsistency between the central and paracentral areas of the cornea $[3,4]$. From the third generation of aspheric IOL, corneal Q value modulation has been involved, which means that according to the corneal $Q$ value under different cornea diameters, high-order aberrations such as spherical aberration, coma, clover-leaf can be adjusted to increase the focal depth of the IOL and correct the visual quality effects caused by high-order aberrations [5].

Previous studies have shown that corneal Q value is closely related to aberrations. Ji C $\mathrm{He}$, et al. [6] found that asphericity (Q) is an important factor leading to the peripheral wavefront aberration through the model eye. The change of corneal $Q$ value has a great impact on high-order aberrations such as spherical aberrations and low-order aberrations (for example, astigmatism) [7]. The corneal $Q$ value is closely related to the refractive state after cataract surgery, through preoperative IOL degree calculation, Woong-Joo Whang, et al. [8] took corneal $\mathrm{Q}$ value modulation into consideration of Haigis-L formula and the diopter prediction error of Haigis-L formula was reduced by about $0.2 \mathrm{D}$. In addition, the spherical aberration at $8.0 \mathrm{~mm}$ cornea diameter was significantly related with the diopter prediction error. Therefore, the corneal Q value was considered in the formula to further reduce the possibility of obvious diopter drift and spherical aberration after cataract surgery. The corneal Q value changes after surgery due to the influence of cataract surgery on the cornea, Giacomo Savini, et al. [9] studied 115 eyes that underwent cataract surgery and found that the greater the absolute value of negative corneal $Q$ value, the more myopia drift occurred after surgery. On the contrary, the larger the positive $Q$ value, the more hyperopia drift arise after surgery. In conclusion, corneal Q value is closely related to postoperative visual acuity and visual quality [10].

The incision position of cataract surgery is a key factor in determining the astigmatism after cataract surgery [11]. However, the influence of incision position on the postoperative corneal $\mathrm{Q}$ value is still unclear. Therefore, we hope to study the influence of different incision positions on corneal $\mathrm{Q}$ value. A retrospective study was conducted on patients using monofocal IOL ZCB00 and trifocal IOL Zeiss 839 who underwent different positions of limbal incision according to the steep meridian. This study analyzed the preoperative, 1-week, and 1-month postoperative corneal $Q$ value and the changes in magnitude to further clarify the trend of corneal $\mathrm{Q}$ value under different incision positions. This study sought to provide a theoretical basis for the relationship between postoperative corneal $Q$ value and visual quality and to provide technical guidance to operators in refractive cataract surgery.

\section{PATIENTS AND METHODS}

This is a retrospective, non-randomized controlled clinical study. All patients were cataract patients who treated Peking University Third Hospital from May 2020 to October 2020. The study was in accordance with the tenets of the Declaration of Helsinki and has been approved by the local ethics committee. Written informed consent was obtained from all patients.

\section{INCLUSION AND EXCLUSION CRITERIAS}

Inclusion criterias: (1)diagnosis of age-related cataract with lens opacity grading greater $\mathrm{C} 2 \mathrm{~N} 2 \mathrm{P0}$ according to the Lens Opacities Classification System III scale; (2) nuclear opacity, cortical opacity, posterior subcapsular opacity, and other lens opacity that significantly affected vision; (3)corneal astigmatism is less than $0.75 \mathrm{D}$.

Exclusion criterias: (1)preoperative severe ocular surface diseases, such as meibomian gland dysfunction, entropion, ectropion, trichiasis, keratopathy; (2)previous eye surgery history, such as silicone oil eye, acute angle-closure glaucoma, glaucoma visual field defect, and other systemic diseases; (3) patients with corneal injury, zonule relaxation, posterior capsule rupture and other complications during the surgery; (4)anterior vitrectomy or intraocular lens suspension; (5)posterior capsular opacity and obvious IOL tilt or decentration during the follow-up; (6)inaccuracy of 
kappa data during corneal topography examination was also excluded.

\section{PREOPERATIVE AND POSTOPERATIVE ASSESSMENT}

A complete preoperative ophthalmological examination was performed on all patients within 1 week before surgery, including distance and near visual acuity, intraocular pressure (IOP), slit-lamp examination, IOL Master (Carl Zeiss Meditec AG, Jena, Germany), Pentacam HR (Oculus Optikgeräte GmbH, Wetzlar, Germany), Oculus (Oculus, Wetzlar, Germany), B-ultrasound, retinal optical coherence tomography (Cirrus 4000; Carl Zeiss Meditec AG, Jena, Germany). And postoperative assessments including visual acuity, slit-lamp examination and Pentacam were performed at 1-week and at 1-month follow up. All ocular corneal Q vlaue were measured by Pentacam. All patients were treated with sodium hyaluronate eye drops before the surgery. Under the cooperation of all patients and stable tear film conditions, the corneal $Q$ value was measured several times, and a stable value was obtained for the study.

\section{SURGICAL PROCEDURE}

All operations were performed by the same experienced surgeon from Peking University Third Hospital under topical anesthesia (Benoxinate $0.4 \%$ solution, 3 times) using a standard phacoemulsification technique. A $3.0 \mathrm{~mm}$ clear corneoscleral incision was made according to the steep axis of the patient's corneal astigmatism, and a lateral incision was made at an angle of $90^{\circ}$ from the main incision, then performed a continuous a $6 \mathrm{~mm}$ circular capsulorhexis, conducted the water separation, carried out the phacoemulsification of cataracts through the main incision, and proceeded automatic suction and perfusion, polished the cortex, afterwards, an intraocular lens was implanted into the capsular bag without additional treatment of the incision. Antibiotic (Cravit 0.5\%), steroidal anti-inflammatory drugs (PredForte 1\%), nonsteroidal antiinflammatory drugs (Pranopulin 1\%)

\section{CORNEAL Q VALUE}

The pre- and post- operative corneal Q value was measured by an experienced technician through Pentacam.

\section{CASE GROUPING}

We used a slitlamp examination was performed with the patient in a sitting position to determine the incision location. Eyes with 12 o'clock incisions were assigned to the superior incision group (ZCB00: 30, Zeiss 839: 17); right eyes with 8 to 10 o'clock (ZCB00: 4, Zeiss 839: 10) and left eyes with 2 to 4 o'clock (ZCB00: 1, Zeiss 839: 4) were assigned to the temporal incision group; right eyes with 2 to 4 o'clock (ZCB00: 1, Zeiss 839: 2) and left eyes with 8 to 10 o'clock (ZCB00: 4, Zeiss 839: 8) were assigned to the nasal incision group, both the temporal and the nasal incisions were horizontal incisions; right eyes with 10 to 12 o'clock (ZCB00: 8, Zeiss 839: 10) and left eyes with 12 to 2 o'clock (ZCB00: 7, Zeiss 839: 10) were assigned to the temporal arc incision group; right eyes with 12 to 2 o'clock (ZCB00: 12, Zeiss 839: 8) and left eyes with 10 to 12 o'clock (ZCB00: 8, Zeiss 839: 14) were assigned to the nasal arc incision group.

\section{STATISTICAL ANALYSIS}

Data are presented as mean \pm standard deviation.

All of the statistical analyses were performed using SPSS 24.0 software (IBM, USA). A paired t test was used to compare variables including the baseline characteristics of patients, the preoperative and postoperative corneal $Q$ value. For normal distribution data, one-way ANOVA was used; for nonnormal distribution data, rank sum test was used. Statistical significance was set at $\mathrm{P}<0.05$.

\section{RESULTS}

We finally analyzed 158 eyes of 103 patients, and the demographics of the study population and the number of eyes included in each group are summarized in Table 1. In 158 eyes of cataract surgery, two types of intraocular lenses Tecnis® ZCB00 (Abbott Medical Optics, Santa Ana, CA) (75 eyes) and AT LISA tri Zeiss 839 (Carl Zeiss Meditec AG, Jena, Germany) (83 eyes) were used. There was no statistically significant difference in baseline parameters between patients with ZCB00 IOL implanted and Zeiss 839 IOL implanted. The data of the five groups of patients with different surgical incisions were all comparable (Table 1). 
Table 1: Baseline characteristics of patients.

\begin{tabular}{|c|c|c|c|c|c|c|}
\hline Items & IOL types & superior incision & nasal incision & temporal incision & $\begin{array}{l}\text { nasal arc } \\
\text { incision }\end{array}$ & $\begin{array}{c}\text { temporal } \\
\text { arc incision }\end{array}$ \\
\hline \multirow[t]{3}{*}{ Eyes (n) } & ZCB00 & 30 & 5 & 5 & 20 & 15 \\
\hline & Zeiss 839 & 17 & 10 & 14 & 22 & 20 \\
\hline & Total & 47 & 15 & 19 & 42 & 35 \\
\hline \multirow[t]{3}{*}{ Male (n) } & ZCB00 & 13 & 0 & 1 & 6 & 5 \\
\hline & Zeiss 839 & 6 & 3 & 5 & 9 & 12 \\
\hline & Total & 19 & 3 & 6 & 15 & 17 \\
\hline \multirow[t]{3}{*}{ Female (n) } & ZCB00 & 17 & 5 & 4 & 13 & 11 \\
\hline & Zeiss 839 & 11 & 7 & 9 & 13 & 8 \\
\hline & Total & 29 & 12 & 13 & 26 & 19 \\
\hline \multirow[t]{3}{*}{ Mean age (y) } & ZCB00 & $69.39 \pm 9.67$ & $66.20 \pm 11.28$ & $62.00 \pm 15.25$ & $64.63 \pm 11.84$ & $66.38 \pm 9.70$ \\
\hline & Zeiss 839 & $61.11 \pm 13.38$ & $68.20 \pm 9.87$ & $73.07 \pm 9.41$ & $63.14 \pm 12.75$ & $62.95 \pm 8.73$ \\
\hline & Total & $66.46 \pm 11.69$ & $67.53 \pm 10.00$ & $70.16 \pm 11.86$ & $63.83 \pm 12.20$ & $64.47 \pm 9.20$ \\
\hline $\begin{array}{c}\text { Nuclear } \\
\text { opalescence } \\
\text { grade } \\
\text { segment }\end{array}$ & Total & $2-4$ & $2-4$ & $2-4$ & $2-4$ & $2-4$ \\
\hline \multirow{3}{*}{$\begin{array}{c}\text { Mean } \\
\text { corneal } \\
\text { astigmatism }\end{array}$} & ZCB00 & $0.60 \pm 0.42$ & $1.24 \pm 1.26$ & $1.10 \pm 0.68$ & $1.31 \pm 1.72$ & $0.80 \pm 0.37$ \\
\hline & Zeiss 839 & $0.81 \pm 0.77$ & $1.03 \pm 0.30$ & $0.96 \pm 0.57$ & $0.82 \pm 0.40$ & $0.59 \pm 0.56$ \\
\hline & Total & $0.67 \pm 0.57$ & $1.11 \pm 0.74$ & $0.96 \pm 0.58$ & $1.06 \pm 1.24$ & $0.89 \pm 0.51$ \\
\hline \multirow{3}{*}{$\begin{array}{l}\text { Mean } \\
\text { corneal } \\
\text { refractive } \\
\text { power }\end{array}$} & ZCB00 & $44.30 \pm 1.00$ & $43.92 \pm 0.86$ & $43.78 \pm 1.35$ & $44.36 \pm 1.58$ & $45.53 \pm 1.37$ \\
\hline & Zeiss 839 & $44.27 \pm 1.76$ & $43.28 \pm 0.73$ & $44.12 \pm 1.49$ & $44.37 \pm 1.37$ & $43.68 \pm 1.03$ \\
\hline & Total & $44.29 \pm 1.31$ & $43.51 \pm 0.81$ & $44.06 \pm 1.39$ & $44.37 \pm 1.45$ & $44.47 \pm 1.49$ \\
\hline
\end{tabular}

We measured the corneal $Q$ factor of all patients before and after the surgery (Table 2, 3). Therefore, this preoperatively and at 1 week and 1 month postoperatively study compared the preoperative and postoperative corneal in all eyes. The statistics showed that the IOL type had no Q factor in the ZCB00, Zeiss 839, and ZCB00 + Zeiss 839 effect on the corneal Q factor of different corneal diameters groups, respectively.

Table 2: The $Q$ value in $6 \mathrm{~mm}$ in the two IOL groups (mean \pm SD).

\begin{tabular}{|c|c|c|c|c|c|}
\hline Items & & ZCB00 & Zeiss 839 & P value & ZCB00+Zeiss 839 \\
\hline \multirow{3}{*}{ Preoperative Q } & Nas & $-0.27 \pm 0.38$ & $-0.08 \pm 0.16$ & 0.998 & $-0.27 \pm 0.33$ \\
\cline { 2 - 6 } & Tem & $-0.08 \pm 0.16$ & $-0.07 \pm 0.13$ & 0.679 & $-0.08 \pm 0.14$ \\
\cline { 2 - 6 } & Inf & $-0.14 \pm 0.29$ & $-0.11 \pm 0.25$ & 0.405 & $-0.13 \pm 0.27$ \\
\cline { 2 - 6 } & Sup & $-0.41 \pm 0.38$ & $-0.25 \pm 0.39$ & 0.01 & $-0.32 \pm 0.40$ \\
\cline { 2 - 6 } & Mean & $-0.23 \pm 0.19$ & $-0.17 \pm 0.18$ & 0.043 & $-0.20 \pm 0.19$ \\
\hline \multirow{3}{*}{ 1 week postoperative Q } & Nas & $-0.35 \pm 0.32$ & $-0.28 \pm 0.29$ & 0.239 & $-0.31 \pm 0.30$ \\
\cline { 2 - 6 } & Tem & $-0.11 \pm 0.13$ & $-0.08 \pm 0.14$ & 0.221 & $-0.09 \pm 0.14$ \\
\cline { 2 - 6 } & Sup & $-0.18 \pm 0.43$ & $-0.15 \pm 0.25$ & 0.547 & $-0.17 \pm 0.34$ \\
\cline { 2 - 6 } & Inf & $-0.50 \pm 0.36$ & $-0.37 \pm 0.56$ & 0.133 & $-0.43 \pm 0.48$ \\
\cline { 2 - 6 } & Mean & $-0.29 \pm 0.17$ & $-0.22 \pm 0.22$ & 0.06 & $-0.25 \pm 0.20$ \\
\hline
\end{tabular}




\begin{tabular}{|c|c|c|c|c|c|}
\hline \multirow{4}{*}{1 month postoperative Q } & Nas & $-0.31 \pm 0.38$ & $-0.24 \pm 0.25$ & 0.233 & $-0.28 \pm 0.32$ \\
\cline { 2 - 6 } & Tem & $-0.11 \pm 0.18$ & $-0.09 \pm 0.14$ & 0.537 & $-0.10 \pm 0.16$ \\
\cline { 2 - 6 } & Inf & $-0.12 \pm 0.27$ & $-0.10 \pm 0.22$ & 0.693 & $-0.11 \pm 0.25$ \\
\cline { 2 - 6 } & Sup & $-0.38 \pm 0.34$ & $-0.39 \pm 0.37$ & 0.863 & $-0.39 \pm 0.35$ \\
\cline { 2 - 6 } & Mean & $-0.24 \pm 0.18$ & $-0.21 \pm 0.17$ & 0.469 & $-0.22 \pm 0.17$ \\
\hline \multirow{4}{*}{$\begin{array}{c}\text { 1 week postoperative Q } \\
\text {-Preoperative Q }\end{array}$} & Nas & $-0.07 \pm 0.44$ & $-0.02 \pm 0.25$ & 0.402 & $-0.04 \pm 0.35$ \\
\cline { 2 - 6 } & Tem & $-0.04 \pm 0.12$ & $-0.01 \pm 0.13$ & 0.186 & $-0.02 \pm 0.13$ \\
\cline { 2 - 6 } & Inf & $-0.05 \pm 0.43$ & $-0.04 \pm 0.23$ & 0.802 & $-0.04 \pm 0.34$ \\
\cline { 2 - 6 } & Sup & $-0.07 \pm 0.40$ & $-0.12 \pm 0.43$ & 0.484 & $-0.10 \pm 0.42$ \\
\cline { 2 - 6 } & Mean & $-0.03 \pm 0.22$ & $-0.05 \pm 0.14$ & 0.537 & $-0.04 \pm 0.18$ \\
\hline \multirow{2}{*}{$\begin{array}{c}1 \text { month postoperative Q } \\
\text {-Preoperative Q }\end{array}$} & Nas & $-0.10 \pm 0.47$ & $0.03 \pm 0.25$ & 0.066 & $-0.03 \pm 0.38$ \\
\cline { 2 - 6 } & Tem & $-0.05 \pm 0.13$ & $-0.02 \pm 0.12$ & 0.256 & $-0.04 \pm 0.13$ \\
\cline { 2 - 6 } & Inf & $-0.01 \pm 0.20$ & $0.03 \pm 0.25$ & 0.384 & $0.25 \pm 0.23$ \\
\cline { 2 - 6 } & Sup & $0.01 \pm 0.37$ & $-0.12 \pm 0.29$ & 0.044 & $0.29 \pm 0.33$ \\
\cline { 2 - 6 } & Mean & $-0.04 \pm 0.17$ & $-0.04 \pm-00.03$ & 0.734 & $0.10 \pm 0.14$ \\
\hline
\end{tabular}

Table 3: The difference of $\mathrm{Q}$ value in $6 \mathrm{~mm}$ pre- and postoperatively.

\begin{tabular}{|c|c|c|c|c|c|c|c|}
\hline \multicolumn{3}{|c|}{ Items } & Nas & Tem & Inf & Sup & Mean \\
\hline \multirow[t]{16}{*}{ ZCВ00 } & \multirow{4}{*}{$\begin{array}{c}\text { Preoperative Q \& } 1 \text { week } \\
\text { postoperative } Q\end{array}$} & Mean & $0.07 \pm 0.44$ & $-0.04 \pm 0.12$ & $0.05 \pm 0.05$ & $0.06 \pm 0.41$ & $0.05 \pm 0.19$ \\
\hline & & $r$ & 0.255 & 0.621 & 0.301 & 0.370 & 0.377 \\
\hline & & $\mathrm{t}$ & 1.189 & 2.486 & 0.857 & 1.229 & 2.229 \\
\hline & & $P$ value & 0.239 & 0.016 & 0.395 & 0.224 & 0.03 \\
\hline & \multirow{4}{*}{$\begin{array}{c}\text { Preoperative } \mathrm{Q} \& 1 \text { month } \\
\text { postoperative } \mathrm{Q}\end{array}$} & Mean & $0.09 \pm 0.47$ & $0.05 \pm 0.13$ & $0.20 \pm 0.03$ & $-0.02 \pm 0.36$ & $0.04 \pm 0.18$ \\
\hline & & $\mathrm{r}$ & 0.311 & 0.731 & 0.765 & 0.526 & 0.562 \\
\hline & & $\mathrm{t}$ & 1.411 & 2.638 & 0.294 & -0.467 & 1.439 \\
\hline & & P value & 0.239 & 0.011 & 0.770 & 0.643 & 0.156 \\
\hline & \multirow{4}{*}{$\begin{array}{c}1 \text { week postoperative } \mathrm{Q} \& 1 \\
\text { month postoperative } \mathrm{Q}\end{array}$} & Mean & $0.00 \pm 0.27$ & $0.01 \pm 0.11$ & $0.51 \pm 0.08$ & $-0.03 \pm 0.32$ & $-0.03 \pm 0.16$ \\
\hline & & $\mathrm{r}$ & 0.677 & 0.637 & 0.331 & 0.585 & 0.557 \\
\hline & & $\mathrm{t}$ & -0.018 & -0.388 & -1.016 & -0.662 & -1.085 \\
\hline & & P value & 0.986 & 0.700 & 0.316 & 0.512 & 0.285 \\
\hline & \multirow{4}{*}{$\begin{array}{c}1 \text { week postoperative } \\
\text { Q -Preoperative Q \& } 1 \\
\text { month postoperative } \mathrm{Q} \\
\text {-Preoperative } \mathrm{Q}\end{array}$} & Mean & $-0.00 \pm 0.27$ & $0.01 \pm 0.11$ & $0.51 \pm 0.08$ & $-0.03 \pm 0.32$ & $0.01 \pm 0.19$ \\
\hline & & $\mathrm{r}$ & 0.867 & 0.629 & 0.250 & 0.730 & 0.661 \\
\hline & & $\mathrm{t}$ & -0.018 & -0.388 & -1.016 & -0.662 & 0.223 \\
\hline & & $P$ value & 0.986 & 0.700 & 0.316 & 0.512 & 0.825 \\
\hline
\end{tabular}




\begin{tabular}{|c|c|c|c|c|c|c|c|}
\hline \multirow{16}{*}{$\begin{array}{c}\text { Zeiss } \\
839\end{array}$} & \multirow{4}{*}{$\begin{array}{c}\text { Preoperative } \mathrm{Q} \& 1 \text { week } \\
\text { postoperative } \mathrm{Q}\end{array}$} & Mean & $0.03 \pm 0.25$ & $0.01 \pm 0.13$ & $0.04 \pm 0.23$ & $0.13 \pm 0.42$ & $0.06 \pm 0.13$ \\
\hline & & $\mathrm{r}$ & 0.635 & 0.545 & 0.591 & 0.652 & 0.808 \\
\hline & & $\mathrm{t}$ & 0.916 & 0.770 & 1.636 & 2.661 & 3.952 \\
\hline & & $P$ value & 0.363 & 0.444 & 0.106 & 0.010 & 0.000 \\
\hline & \multirow{4}{*}{$\begin{array}{c}\text { Preoperative Q \&1 month } \\
\text { postoperative Q }\end{array}$} & Mean & $-0.03 \pm 0.25$ & $0.02 \pm 0.12$ & $-0.03 \pm 0.24$ & $0.13 \pm 0.28$ & $0.09 \pm 0.01$ \\
\hline & & $\mathrm{r}$ & 0.551 & 0.509 & 0.431 & 0.712 & 0.843 \\
\hline & & $\mathrm{t}$ & -0.948 & 1.184 & -0.804 & 3.445 & 2.948 \\
\hline & & $P$ value & 0.347 & 0.242 & 0.425 & 0.001 & 0.005 \\
\hline & \multirow{4}{*}{$\begin{array}{c}1 \text { week postoperative } \mathrm{Q} \& 1 \\
\text { month postoperative } \mathrm{Q}\end{array}$} & Mean & $-0.03 \pm 0.22$ & $0.02 \pm 0.12$ & $-0.03 \pm 0.11$ & $0.02 \pm 0.32$ & $0.00 \pm 0.09$ \\
\hline & & $\mathrm{r}$ & 0.619 & 0.662 & 0.889 & 0.715 & 0.892 \\
\hline & & $\mathrm{t}$ & -0.897 & 1.079 & -1.965 & 0.397 & -0.100 \\
\hline & & $\mathrm{P}$ value & 0.375 & 0.286 & 0.056 & 0.693 & 0.920 \\
\hline & \multirow{4}{*}{$\begin{array}{c}1 \text { week postoperative } \\
\text { Q - Preoperative Q \& } 1 \\
\text { month postoperative } Q \\
\text {-Preoperative } Q\end{array}$} & Mean & $-0.02 \pm 0.22$ & $0.03 \pm 0.12$ & $-0.04 \pm 0.14$ & $0.02 \pm 0.33$ & $0.00 \pm 0.11$ \\
\hline & & $\mathrm{r}$ & 0.620 & 0.549 & 0.859 & 0.504 & 0.556 \\
\hline & & $\mathrm{t}$ & -0.776 & 1.614 & -1.841 & 0.416 & 0.027 \\
\hline & & $\mathrm{P}$ value & 0.442 & 0.113 & 0.072 & 0.679 & 0.979 \\
\hline \multirow{16}{*}{$\begin{array}{c}\text { ZCB } \\
00 \\
+ \text { Zeiss } \\
839\end{array}$} & \multirow{4}{*}{$\begin{array}{c}\text { Preoperative Q \& } 1 \text { week } \\
\text { postoperative } Q\end{array}$} & Mean & $0.04 \pm 0.35$ & $0.02 \pm 0.13$ & $0.05 \pm 0.34$ & $0.10 \pm 0.42$ & $0.06 \pm 0.16$ \\
\hline & & $r$ & 0.419 & 0.577 & 0.404 & 0.565 & 0.657 \\
\hline & & $\mathrm{t}$ & 1.496 & 2.175 & 1.566 & 2.811 & 4.161 \\
\hline & & $\mathrm{P}$ value & 0.137 & 0.031 & 0.120 & 0.006 & 0.000 \\
\hline & \multirow{4}{*}{$\begin{array}{c}\text { Preoperative Q \&1 month } \\
\text { postoperative Q }\end{array}$} & Mean & $0.03 \pm 0.38$ & $0.03 \pm 0.13$ & $-0.01 \pm 0.22$ & $0.06 \pm 0.33$ & $0.04 \pm 0.14$ \\
\hline & & $r$ & 0.376 & 0.650 & 0.626 & 0.608 & 0.695 \\
\hline & & $\mathrm{t}$ & 0.766 & 2.707 & -0.453 & 1.758 & 2.716 \\
\hline & & $\mathrm{P}$ value & 0.445 & 0.008 & 0.651 & 0.082 & 0.008 \\
\hline & \multirow{4}{*}{$\begin{array}{c}1 \text { week postoperative } \mathrm{Q} \& 1 \\
\text { month postoperative } \mathrm{Q}\end{array}$} & Mean & $-0.16 \pm 0.24$ & $0.01 \pm 0.12$ & $-0.06 \pm 0.35$ & $-0.01 \pm 0.32$ & $-0.01 \pm 0.12$ \\
\hline & & $r$ & 0.655 & 0.648 & 0.485 & 0.669 & 0.766 \\
\hline & & $\mathrm{t}$ & -0.617 & 0.569 & -1.449 & -0.152 & -0.977 \\
\hline & & P value & 0.539 & 0.571 & 0.151 & 0.880 & 0.332 \\
\hline & \multirow{4}{*}{$\begin{array}{c}1 \text { week postoperative } \\
\text { Q - Preoperative Q \& } 1 \\
\text { month postoperative } Q \\
\text {-Preoperative } Q\end{array}$} & Mean & $-0.01 \pm 0.24$ & $0.01 \pm 0.12$ & $-0.06 \pm 0.35$ & $-0.00 \pm 0.33$ & $0.00 \pm 0.15$ \\
\hline & & $r$ & 0.811 & 0.586 & 0.474 & 0.628 & 0.635 \\
\hline & & $\mathrm{t}$ & -0.545 & 0.997 & -1.519 & -0.105 & 0.206 \\
\hline & & $P$ value & 0.587 & 0.321 & 0.132 & 0.917 & 0.837 \\
\hline
\end{tabular}

In ZCB00 group, at $6.0 \mathrm{~mm}$ corneal diameter, the temporal corneal Q value was $-0.08 \pm 0.16 \mathrm{~mm}$ preoperatively, $-0.11 \pm 0.13 \mathrm{~mm}$ at 1 week postoperatively and $-0.11 \pm 0.18 \mathrm{~mm}$ at 1 month postoperatively; the mean corneal $Q$ value was $-0.23 \pm 0.19 \mathrm{~mm}$ preoperatively and $-0.29 \pm 0.17 \mathrm{~mm}$ at 1 week postoperatively. The temporal corneal $Q$ value at 1 month postoperatively decreased $0.04 \pm 0.12$ and $0.05 \pm 0.13 \mathrm{~mm}$ compared with it at 1 week postoperatively and preoperatively; the mean corneal $Q$ value at 1 month postoperatively decreased $0.03 \pm 0.22 \mathrm{~mm}$ compared with it preoperatively. Similarly, in the Zeiss 839 group, at
$6.0 \mathrm{~mm}$ corneal diameter, the superior corneal $\mathrm{Q}$ value was $-0.25 \pm 0.39 \mathrm{~mm}$ preoperatively, $-0.37 \pm 0.56 \mathrm{~mm}$ at 1 week postoperatively and $-0.39 \pm 0.37 \mathrm{~mm}$ at 1 month postoperatively; the mean corneal Q value was $-0.17 \pm 0.18 \mathrm{~mm}$ preoperatively, $-0.22 \pm 0.22 \mathrm{~mm}$ at 1 week postoperatively and $-0.21 \pm 0.17 \mathrm{~mm}$ at 1 month postoperatively. The superior and mean corneal $\mathrm{Q}$ value at 1 week postoperatively decreased $0.12 \pm 0.43 \mathrm{~mm}$ and $0.05 \pm 0.14 \mathrm{~mm}$ respectively compared with it preoperatively, the superior and mean corneal $Q$ value at 1 month postoperatively decreased $0.12 \pm 0.29 \mathrm{~mm}$ and $0.04 \pm-$ $00.03 \mathrm{~mm}$ respectively compared with it preoperatively. In 
ZCB00 + Zeiss 839 group, at $6.0 \mathrm{~mm}$ corneal diameter, the temporal corneal $Q$ value was $-0.08 \pm 0.14 \mathrm{~mm}$ preoperatively and $-0.10 \pm 0.16 \mathrm{~mm}$ at 1 month postoperatively; the superior corneal $\mathrm{Q}$ value was $-0.32 \pm 0.40 \mathrm{~mm}$ preoperatively and $-0.43 \pm 0.48 \mathrm{~mm}$ at 1 week postoperatively ; the mean corneal $Q$ value was $-0.20 \pm 0.19 \mathrm{~mm}$ preoperatively, $-0.25 \pm 0.20 \mathrm{~mm}$ at 1 week postoperatively and $-0.22 \pm 0.17 \mathrm{~mm}$ at 1 month postoperatively. The superior and mean corneal $Q$ value at 1 week postoperatively decreased $0.10 \pm 0.42 \mathrm{~mm}$ and $0.04 \pm 0.18 \mathrm{~mm}$ compared with it preoperatively; the temporal and mean corneal $\mathrm{Q}$ value at 1 month postoperatively decreased $0.04 \pm 0.13 \mathrm{~mm}$ and $0.10 \pm 0.14 \mathrm{~mm}$ compared with it preoperatively.

At $7.0 \mathrm{~mm}$ corneal diameter, In ZCB00 group, the temporal corneal Q factor was $-0.13 \pm 0.02 \mathrm{~mm}$ preoperatively, $-0.14 \pm 0.02 \mathrm{~mm}$ at 1 week postoperatively and $-0.16 \pm 0.02 \mathrm{~mm}$ at 1 month postoperatively; the superior corneal $\mathrm{Q}$ factor was $-0.53 \pm 0.04 \mathrm{~mm}$ preoperatively and $-0.50 \pm 0.04 \mathrm{~mm}$ at 1 month postoperatively. The temporal corneal $\mathrm{Q}$ factor decreased $0.03 \pm 0.01 \mathrm{~mm}$ and $0.03 \pm 0.02 \mathrm{~mm}$ at 1 week and 1 month postoperatively compared with it preoperatively; the superior corneal $\mathrm{Q}$ factor at 1 month postoperatively increased $0.04 \pm 0.05 \mathrm{~mm}$ compared with it preoperatively. Similarly, in the Zeiss 839 group, the temporal corneal Q factor was $-0.10 \pm 0.01 \mathrm{~mm}$ preoperatively and $-0.14 \pm 0.02 \mathrm{~mm}$ at 1 month postoperatively; the superior corneal $\mathrm{Q}$ factor was $-0.36 \pm 0.04 \mathrm{~mm}$ preoperatively, $-0.46 \pm 0.06 \mathrm{~mm}$ at 1 week postoperatively and $-0.48 \pm 0.04 \mathrm{~mm}$ at 1 month postoperatively; the mean corneal $Q$ factor was $-0.29 \pm 0.02 \mathrm{~mm}$ preoperatively, $-0.29 \pm 0.02 \mathrm{~mm}$ at 1 week postoperatively and $-0.48 \pm 0.04 \mathrm{~mm}$ at 1 month postoperatively. The superior and mean corneal $\mathrm{Q}$ factor at 1 week postoperatively decreased $0.09 \pm 0.04 \mathrm{~mm}$ and $0.04 \pm 0.01 \mathrm{~mm}$ respectively compared with it preoperatively; the temporal, superior and mean corneal Q factor at 1 month postoperatively decreased $0.04 \pm 0.02 \mathrm{~mm}$, $0.09 \pm 0.04 \mathrm{~mm}$ and $0.02 \pm 0.01 \mathrm{~mm}$ respectively compared with it preoperatively. In ZCB00 + Zeiss 839 group, the temporal corneal $\mathrm{Q}$ factor was $-0.09 \pm 0.01 \mathrm{~mm}$ preoperatively and $-0.11 \pm 0.01 \mathrm{~mm}$ at 1 month postoperatively; the superior corneal $\mathrm{Q}$ factor was $-0.35 \pm 0.03 \mathrm{~mm}$ preoperatively and $-0.45 \pm 0.04 \mathrm{~mm}$ at 1 week postoperatively; the mean corneal $\mathrm{Q}$ factor was $-0.21 \pm 0.02 \mathrm{~mm}$ preoperatively, $-0.26 \pm 0.02 \mathrm{~mm}$ at 1 week postoperatively and $-0.22 \pm 0.17 \mathrm{~mm}$ at 1 month postoperatively. The superior and mean corneal $Q$ factor at 1 week postoperatively decreased $0.10 \pm 0.42 \mathrm{~mm}$ and $-0.25 \pm 0.02 \mathrm{~mm}$ compared with it preoperatively; the temporal and mean corneal $\mathrm{Q}$ factor at 1 month postoperatively decreased $0.03 \pm 0.01 \mathrm{~mm}$ and $0.04 \pm 0.01 \mathrm{~mm}$ compared with it preoperatively.
At $8.0 \mathrm{~mm}$ corneal diameter, In ZCB00 group, the temporal corneal Q value was $0.19 \pm 0.02 \mathrm{~mm}$ preoperatively and $-0.22 \pm 0.03 \mathrm{~mm}$ at 1 month postoperatively. The temporal corneal $Q$ value decreased $0.04 \pm 0.02 \mathrm{~mm}$ at 1 month postoperatively compared with it preoperatively. Similarly, in the Zeiss 839 group, the temporal corneal $\mathrm{Q}$ value was $-0.14 \pm 0.01 \mathrm{~mm}$ preoperatively, $-0.17 \pm 0.02$ $\mathrm{mm}$ at 1 week postoperatively and $-0.20 \pm 0.20 \mathrm{~mm}$ at 1 month postoperatively; the inferior corneal $Q$ value was $-0.23 \pm 0.02 \mathrm{~mm}$ preoperatively and $-0.27 \pm 0.03 \mathrm{~mm}$ at 1 week postoperatively; the superior corneal $Q$ value was $-0.49 \pm 0.04 \mathrm{~mm}$ preoperatively and $0.56 \pm 0.04 \mathrm{~mm}$ at 1 month postoperatively; the mean corneal $\mathrm{Q}$ value was $-0.33 \pm 0.02 \mathrm{~mm}$ preoperatively and $-0.36 \pm 0.02 \mathrm{~mm}$ at 1 week postoperatively. The temporal, inferior and mean corneal $Q$ value at 1 week postoperatively decreased $0.04 \pm 0.02 \mathrm{~mm}$, $0.05 \pm 0.02 \mathrm{~mm}$ and $0.03 \pm 0.02 \mathrm{~mm}$ respectively compared with it preoperatively; the temporal and superior corneal $\mathrm{Q}$ value at 1 month postoperatively decreased $0.06 \pm 0.02$ $\mathrm{mm}$ and $0.04 \pm 0.05 \mathrm{~mm}$ respectively compared with it preoperatively. In ZCB00 + Zeiss 839 group, the temporal corneal $Q$ value was $-0.16 \pm 0.01 \mathrm{~mm}$ preoperatively, $-0.18 \pm 0.01 \mathrm{~mm}$ at 1 week postoperatively and $-0.21 \pm 0.02$ $\mathrm{mm}$ at 1 month postoperatively; the inferior corneal $\mathrm{Q}$ value was $-0.26 \pm 0.02 \mathrm{~mm}$ preoperatively and $-0.29 \pm 0.03 \mathrm{~mm}$ at 1 week postoperatively. The temporal and inferior corneal $\mathrm{Q}$ value at 1 week postoperatively decreased $0.03 \pm 0.01$ $\mathrm{mm}$ and $0.05 \pm 0.02 \mathrm{~mm}$ compared with it preoperatively; the temporal corneal $\mathrm{Q}$ value at 1 month postoperatively decreased $0.05 \pm 0.01 \mathrm{~mm}$ compared with it preoperatively.

At $9.0 \mathrm{~mm}$ corneal diameter, In ZCB00 group, there was no statistically significant difference in corneal $Q$ value at 1 week and 1 month postoperatively compared with the preoperative corneal $\mathrm{Q}$ factor. In the Zeiss 839 group, the temporal corneal Q value was $-0.21 \pm 0.02 \mathrm{~mm}$ preoperatively, $-0.24 \pm 0.02 \mathrm{~mm}$ at 1 week postoperatively and $-0.28 \pm 0.02$ $\mathrm{mm}$ at 1 month postoperatively; the inferior corneal $\mathrm{Q}$ value was $-0.37 \pm 0.02 \mathrm{~mm}$ preoperatively and $-0.30 \pm 0.03 \mathrm{~mm}$ at 1 month postoperatively. The temporal corneal $\mathrm{Q}$ value at 1 week postoperatively decreased $0.06 \pm 0.02 \mathrm{~mm}$ compared with it preoperatively; the temporal corneal $Q$ value at 1 month postoperatively decreased $0.08 \pm 0.03 \mathrm{~mm}$ and the inferior corneal $Q$ value increased $0.07 \pm 0.03 \mathrm{~mm}$ compared with it preoperatively. In ZCB00 + Zeiss 839 group, the temporal corneal $Q$ value was $-0.22 \pm 0.01 \mathrm{~mm}$ preoperatively, $-0.25 \pm 0.01 \mathrm{~mm}$ at 1 week postoperatively and $-0.29 \pm 0.02$ $\mathrm{mm}$ at 1 month postoperatively. The temporal corneal $\mathrm{Q}$ value decreased $0.03 \pm 0.01 \mathrm{~mm}$ and $0.07 \pm 0.02 \mathrm{~mm}$ at 1 week and 1 month postoperatively respectively compared with 
it preoperatively. The temporal corneal $\mathrm{Q}$ value at 1 week $(\mathrm{P}=0.015)$ and 1 month $(\mathrm{P}=0.002)$ postoperatively significantly decreased compared with that preoperatively.

At $10.0 \mathrm{~mm}$ corneal diameter, In ZCB00 group, the superior corneal $Q$ value was $-0.72 \pm 0.03 \mathrm{~mm}$ preoperatively and $-0.68 \pm 0.03 \mathrm{~mm}$ at 1 month postoperatively. The superior corneal $Q$ value increased $0.07 \pm 0.04 \mathrm{~mm}$ at 1 month postoperatively compared with it preoperatively. Similarly, in the Zeiss 839 group, the temporal corneal $Q$ value was $-0.29 \pm 0.02 \mathrm{~mm}$ preoperatively and $-0.35 \pm 0.03 \mathrm{~mm}$ at 1 month postoperatively. The temporal corneal $\mathrm{Q}$ value at 1 month postoperatively decreased $0.07 \pm 0.03 \mathrm{~mm}$ compared with it preoperatively. In ZCB00 + Zeiss 839 group, the temporal corneal $Q$ value was $-0.30 \pm 0.01 \mathrm{~mm}$ preoperatively and $-0.35 \pm 0.02 \mathrm{~mm}$ at 1 month postoperatively; the superior corneal $Q$ value was $-0.72 \pm 0.02 \mathrm{~mm}$ preoperatively and $-0.66 \pm 0.02 \mathrm{~mm}$ at 1 month postoperatively. The temporal corneal $Q$ value decreased $0.02 \pm 0.01 \mathrm{~mm}$ and $0.06 \pm 0.02 \mathrm{~mm}$ at 1 week and 1 month postoperatively compared with it preoperatively respectively.

Table 4: The $Q$ value in $7 \mathrm{~mm}$ in the two IOL groups (mean $\pm \mathrm{SD}$ ).

\begin{tabular}{|c|c|c|c|c|}
\hline \multicolumn{2}{|l|}{ Items } & ZCB00 & Zeiss 839 & ZCB00+Zeiss 839 \\
\hline \multirow[t]{5}{*}{ Preoperative Q } & Nas & $-0.41 \pm 0.05$ & $-0.36 \pm 0.04$ & $-0.30 \pm 0.03$ \\
\hline & Tem & $-0.13 \pm 0.02$ & $-0.10 \pm 0.01$ & $-0.09 \pm 0.01$ \\
\hline & Inf & $-0.21 \pm 0.03$ & $-0.15 \pm 0.02$ & $-0.14 \pm 0.02$ \\
\hline & Sup & $-0.53 \pm 0.04$ & $-0.36 \pm 0.04$ & $-0.35 \pm 0.03$ \\
\hline & Mean & $-0.32 \pm 0.02$ & $-0.25 \pm 0.02$ & $-0.21 \pm 0.02$ \\
\hline \multirow[t]{5}{*}{1 week postoperative $Q$} & Nas & $-0.46 \pm 0.05$ & $-0.39 \pm 0.03$ & $-0.34 \pm 0.03$ \\
\hline & Tem & $-0.14 \pm 0.02$ & $-0.12 \pm 0.02$ & $-0.10 \pm 0.01$ \\
\hline & Inf & $-0.25 \pm 0.05$ & $-0.19 \pm 0.03$ & $-0.19 \pm 0.03$ \\
\hline & Sup & $-0.58 \pm 0.04$ & $-0.46 \pm 0.06$ & $-0.45 \pm 0.04$ \\
\hline & Mean & $-0.35 \pm 0.03$ & $-0.29 \pm 0.02$ & $-0.26 \pm 0.02$ \\
\hline \multirow[t]{5}{*}{1 month postoperative $Q$} & Nas & $-0.45 \pm 0.05$ & $-0.34 \pm 0.03$ & $-0.31 \pm 0.03$ \\
\hline & Tem & $-0.16 \pm 0.02$ & $-0.14 \pm 0.02$ & $-0.11 \pm 0.01$ \\
\hline & Inf & $-0.21 \pm 0.03$ & $-0.15 \pm 0.03$ & $-0.13 \pm 0.02$ \\
\hline & Sup & $-0.50 \pm 0.04$ & $-0.48 \pm 0.04$ & $-0.41 \pm 0.03$ \\
\hline & Mean & $-0.33 \pm 0.02$ & $-0.28 \pm 0.02$ & $-0.25 \pm 0.02$ \\
\hline \multirow[t]{5}{*}{1 week postoperative $Q$-Preoperative $Q$} & Nas & $-0.03 \pm 0.06$ & $-0.01 \pm 0.03$ & $-0.04 \pm 0.03$ \\
\hline & Tem & $-0.03 \pm 0.01$ & $-0.02 \pm 0.01$ & $-0.02 \pm 0.01$ \\
\hline & Inf & $-0.06 \pm 0.05$ & $-0.05 \pm 0.02$ & $-0.04 \pm 0.03$ \\
\hline & Sup & $-0.03 \pm 0.05$ & $-0.09 \pm 0.04$ & $-0.09 \pm 0.04$ \\
\hline & Mean & $-0.03 \pm 0.03$ & $-0.04 \pm 0.01$ & $-0.04 \pm 0.01$ \\
\hline \multirow{5}{*}{$\begin{array}{c}1 \text { month postoperative } \mathrm{Q} \text {-Preoperative } \\
\mathrm{Q}\end{array}$} & Nas & $-0.06 \pm 0.06$ & $-0.00 \pm 0.03$ & $-0.03 \pm 0.04$ \\
\hline & Tem & $-0.03 \pm 0.02$ & $-0.04 \pm 0.02$ & $-0.03 \pm 0.01$ \\
\hline & Inf & $-0.03 \pm 0.03$ & $0.03 \pm 0.03$ & $0.00 \pm 0.02$ \\
\hline & Sup & $0.04 \pm 0.05$ & $-0.09 \pm 0.04$ & $-0.05 \pm 0.03$ \\
\hline & Mean & $-0.02 \pm 0.02$ & $-0.02 \pm 0.01$ & $-0.04 \pm 0.01$ \\
\hline
\end{tabular}


Table 5: The difference of Q value in $7 \mathrm{~mm}$ pre- and postoperatively.

\begin{tabular}{|c|c|c|c|c|c|c|c|}
\hline \multicolumn{3}{|c|}{ Items } & Nas & Tem & Inf & Sup & Mean \\
\hline \multirow[t]{16}{*}{ ZCB00 } & \multirow{4}{*}{$\begin{array}{c}\text { Preoperative Q \& } 1 \text { week } \\
\text { postoperative Q }\end{array}$} & Mean & $0.03 \pm 0.47$ & $0.03 \pm 0.11$ & $0.06 \pm 0.40$ & $0.42 \pm 0.05$ & $0.03 \pm 0.21$ \\
\hline & & $r$ & 0.317 & 0.668 & 0.276 & 0.356 & 0.397 \\
\hline & & $\mathrm{t}$ & 0.555 & 2.185 & 1.151 & 0.469 & 0.977 \\
\hline & & $P$ value & 0.581 & 0.033 & 0.254 & 0.640 & 0.333 \\
\hline & \multirow{4}{*}{$\begin{array}{l}\text { Preoperative Q \&1 month } \\
\text { postoperative } Q\end{array}$} & Mean & $0.07 \pm 0.46$ & $0.04 \pm 0.11$ & $0.03 \pm 0.21$ & $0.36 \pm 0.05$ & $0.03 \pm 0.16$ \\
\hline & & $\mathrm{r}$ & 0.393 & 0.787 & 0.598 & 0.284 & 0.549 \\
\hline & & $\mathrm{t}$ & 1.172 & 2.740 & 1.078 & -4.088 & 1.305 \\
\hline & & $P$ value & 0.246 & 0.008 & 0.286 & 0.000 & 0.197 \\
\hline & \multirow{4}{*}{$\begin{array}{c}1 \text { week postoperative } Q \\
\& 1 \text { month postoperative } \\
Q\end{array}$} & Mean & $0.03 \pm 0.19$ & $0.00 \pm 0.11$ & $-0.06 \pm 0.45$ & $0.20 \pm 0.03$ & $-0.01 \pm 0.20$ \\
\hline & & $\mathrm{r}$ & 0.864 & 0.610 & 0.267 & 0.391 & 0.391 \\
\hline & & $\mathrm{t}$ & 0.986 & 0.043 & -0.897 & -0.470 & -0.470 \\
\hline & & $P$ value & 0.330 & 0.966 & 0.375 & 0.641 & 0.641 \\
\hline & \multirow{4}{*}{$\begin{array}{c}1 \text { week postoperative } \\
\text { Q -Preoperative Q \& } 1 \\
\text { month postoperative Q } \\
\text {-Preoperative } Q\end{array}$} & Mean & $0.01 \pm 0.24$ & $-0.01 \pm 0.11$ & $-0.07 \pm 0.45$ & $0.44 \pm 0.07$ & $-0.02 \pm 0.20$ \\
\hline & & r & 0.884 & 0.508 & 0.265 & 0.308 & 0.512 \\
\hline & & $\mathrm{t}$ & 0.167 & -0.312 & -0.961 & -0.288 & -0.762 \\
\hline & & $P$ value & 0.868 & 0.757 & 0.342 & 0.775 & 0.450 \\
\hline \multirow[t]{16}{*}{ Zeiss 839} & \multirow{4}{*}{$\begin{array}{c}\text { Preoperative Q \& } 1 \text { week } \\
\text { postoperative Q }\end{array}$} & Mean & $0.01 \pm 0.27$ & $0.02 \pm 0.13$ & $0.04 \pm 0.21$ & $0.10 \pm 0.37$ & $0.04 \pm 0.12$ \\
\hline & & $\mathrm{r}$ & 0.631 & 0.475 & 0.618 & 0.654 & 0.819 \\
\hline & & $\mathrm{t}$ & 0.292 & 1.510 & 1.665 & 2.248 & 2.833 \\
\hline & & $P$ value & 0.771 & 0.135 & 0.100 & 0.028 & 0.006 \\
\hline & \multirow{4}{*}{$\begin{array}{l}\text { Preoperative } \mathrm{Q} \& 1 \text { month } \\
\text { postoperative } \mathrm{Q}\end{array}$} & Mean & $0.00 \pm 0.24$ & $0.04 \pm 0.13$ & $-0.02 \pm 0.20$ & $0.10 \pm 0.31$ & $0.03 \pm 0.09$ \\
\hline & & $\mathrm{r}$ & 0.633 & 0.419 & 0.505 & 0.683 & 0.862 \\
\hline & & $\mathrm{t}$ & 0.140 & 2.520 & -0.797 & 2.280 & 2.601 \\
\hline & & $P$ value & 0.889 & 0.015 & 0.429 & 0.026 & 0.012 \\
\hline & \multirow{4}{*}{$\begin{array}{c}1 \text { week postoperative } \mathrm{Q} \\
\& 1 \text { month postoperative } \\
\mathrm{Q}\end{array}$} & Mean & $0.01 \pm 0.13$ & $0.02 \pm 0.11$ & $-0.05 \pm 0.11$ & $0.01 \pm 0.35$ & $0.00 \pm 0.09$ \\
\hline & & $\mathrm{r}$ & 0.832 & 0.694 & 0.880 & 0.639 & 0.873 \\
\hline & & $\mathrm{t}$ & 0.760 & 0.988 & -2.859 & 0.267 & 0.032 \\
\hline & & P value & 0.451 & 0.329 & 0.006 & 0.790 & 0.975 \\
\hline & \multirow{4}{*}{$\begin{array}{c}1 \text { week postoperative } \\
\text { Q -Preoperative Q \& } 1 \\
\text { month postoperative Q } \\
\text {-Preoperative Q }\end{array}$} & Mean & $0.01 \pm 0.13$ & $0.02 \pm 0.11$ & $-0.05 \pm 0.11$ & $0.13 \pm 0.35$ & $0.00 \pm 0.09$ \\
\hline & & $\mathrm{r}$ & 0.884 & 0.623 & 0.863 & 0.495 & 0.520 \\
\hline & & $\mathrm{t}$ & 0.760 & 0.988 & -2.859 & 0.267 & 0.032 \\
\hline & & $P$ value & 0.451 & 0.329 & 0.006 & 0.790 & 0.975 \\
\hline
\end{tabular}




\begin{tabular}{|c|c|c|c|c|c|c|c|}
\hline \multirow{16}{*}{$\begin{array}{l}\text { ZCB00 } \\
+ \text { Zeiss } \\
839\end{array}$} & \multirow{4}{*}{$\begin{array}{c}\text { Preoperative Q \& } 1 \text { week } \\
\text { postoperative } Q\end{array}$} & Mean & $0.03 \pm 0.37$ & $0.02 \pm 0.13$ & $0.04 \pm 0.36$ & $0.08 \pm 0.44$ & $0.04 \pm 0.18$ \\
\hline & & $\mathrm{r}$ & 0.413 & 0.536 & 0.303 & 0.524 & 0.597 \\
\hline & & $\mathrm{t}$ & 1.083 & 1.569 & 1.300 & 2.211 & 2.703 \\
\hline & & $P$ value & 0.281 & 0.119 & 0.196 & 0.029 & 0.008 \\
\hline & \multirow{4}{*}{$\begin{array}{c}\text { Preoperative } \mathrm{Q} \& 1 \text { month } \\
\text { postoperative } \mathrm{Q}\end{array}$} & Mean & $0.03 \pm 0.38$ & $0.03 \pm 0.12$ & $-0.05 \pm 0.36$ & $0.06 \pm 0.34$ & $0.04 \pm 0.13$ \\
\hline & & $\mathrm{r}$ & 0.435 & 0.652 & 0.402 & 0.581 & 0.727 \\
\hline & & $\mathrm{t}$ & 0.836 & 2.768 & -1.271 & 1.783 & 3.049 \\
\hline & & $P$ value & 0.405 & 0.007 & 0.207 & 0.077 & 0.003 \\
\hline & \multirow{4}{*}{$\begin{array}{c}1 \text { week postoperative } \mathrm{Q} \\
\& 1 \text { month postoperative } \\
\mathrm{Q}\end{array}$} & Mean & $-0.00 \pm 0.21$ & $-0.08 \pm 0.14$ & $-0.00 \pm 0.23$ & $-0.03 \pm 0.34$ & $-0.01 \pm 0.15$ \\
\hline & & $r$ & 0.773 & 0.471 & 0.545 & 0.636 & 0.696 \\
\hline & & $\mathrm{t}$ & -0.207 & -6.604 & -0.173 & -0.862 & -0.582 \\
\hline & & $P$ value & 0.836 & 0.000 & 0.863 & 0.391 & 0.562 \\
\hline & \multirow{4}{*}{$\begin{array}{c}1 \text { week postoperative } \\
\text { Q -Preoperative Q \& } 1 \\
\text { month postoperative Q } \\
\text {-Preoperative Q }\end{array}$} & Mean & $-0.00 \pm 0.21$ & $-0.00 \pm 0.44$ & $-0.05 \pm 0.35$ & $-0.03 \pm 0.34$ & $-0.01 \pm 0.16$ \\
\hline & & $\mathrm{r}$ & 0.855 & -0.201 & 0.452 & 0.610 & 0.566 \\
\hline & & $\mathrm{t}$ & -0.137 & -0.062 & -1.418 & -0.704 & -0.410 \\
\hline & & P value & 0.891 & 0.950 & 0.160 & 0.483 & 0.683 \\
\hline
\end{tabular}

Table 6: The $Q$ value in $8 \mathrm{~mm}$ in the two IOL groups (mean $\pm \mathrm{SD}$ ).

\begin{tabular}{|c|c|c|c|c|}
\hline Items & & ZCB00 & Zeiss 839 & ZCB00+Zeiss 839 \\
\hline \multirow[t]{5}{*}{ Preoperative Q } & Nas & $-0.54 \pm 0.05$ & $-0.49 \pm 0.04$ & $-0.51 \pm 0.03$ \\
\hline & Tem & $-0.19 \pm 0.02$ & $-0.14 \pm 0.01$ & $-\mathbf{0 . 1 6} \pm 0.01$ \\
\hline & Inf & $-0.30 \pm 0.03$ & $-0.23 \pm 0.02$ & $-0.26 \pm 0.02$ \\
\hline & Sup & $-0.62 \pm 0.04$ & $-0.49 \pm 0.04$ & $-0.55 \pm 0.03$ \\
\hline & Mean & $-0.41 \pm 0.02$ & $-0.33 \pm 0.02$ & $-0.37 \pm 0.02$ \\
\hline \multirow[t]{5}{*}{1 week postoperative $Q$} & Nas & $-1.93 \pm 1.41$ & $-0.48 \pm 0.03$ & $-1.15 \pm 0.65$ \\
\hline & Tem & $-0.19 \pm 0.02$ & $-0.17 \pm 0.02$ & $-0.18 \pm 0.01$ \\
\hline & Inf & $-0.33 \pm 0.04$ & $-0.27 \pm 0.03$ & $-0.29 \pm 0.03$ \\
\hline & Sup & $-0.67 \pm 0.04$ & $-0.52 \pm 0.05$ & $-0.59 \pm 0.04$ \\
\hline & Mean & $-0.41 \pm 0.03$ & $-0.36 \pm 0.02$ & $-0.38 \pm 0.02$ \\
\hline \multirow[t]{5}{*}{1 month postoperative $\mathrm{Q}$} & Nas & $-0.59 \pm 0.06$ & $-0.47 \pm 0.03$ & $-0.52 \pm 0.03$ \\
\hline & Tem & $-0.22 \pm 0.03$ & $-0.20 \pm 0.20$ & $-0.21 \pm 0.02$ \\
\hline & Inf & $-0.33 \pm 0.03$ & $-0.19 \pm 0.03$ & $-0.26 \pm 0.02$ \\
\hline & Sup & $-0.59 \pm 0.04$ & $-0.56 \pm 0.04$ & $-0.57 \pm 0.03$ \\
\hline & Mean & $-0.43 \pm 0.02$ & $-0.36 \pm 0.02$ & $-0.39 \pm 0.02$ \\
\hline \multirow{5}{*}{$\begin{array}{c}1 \text { week postoperative } \mathrm{Q} \\
\text {-Preoperative } \mathrm{Q}\end{array}$} & Nas & $-1.39 \pm 1.41$ & $0.02 \pm 0.03$ & $-0.63 \pm 0.65$ \\
\hline & Tem & $-0.02 \pm 0.01$ & $-0.04 \pm 0.02$ & $-0.03 \pm 0.01$ \\
\hline & Inf & $-0.05 \pm 0.04$ & $-0.05 \pm 0.02$ & $-0.05 \pm 0.02$ \\
\hline & Sup & $-0.02 \pm 0.05$ & $-0.03 \pm 0.05$ & $-0.03 \pm 0.03$ \\
\hline & Mean & $0.00 \pm 0.03$ & $-0.03 \pm 0.02$ & $-0.01 \pm 0.02$ \\
\hline \multirow{5}{*}{$\begin{array}{c}1 \text { month postoperative } \mathrm{Q} \\
\text {-Preoperative } \mathrm{Q}\end{array}$} & Nas & $-0.06 \pm 0.06$ & $-0.00 \pm 0.03$ & $-0.03 \pm 0.03$ \\
\hline & Tem & $-0.04 \pm 0.02$ & $-0.06 \pm 0.02$ & $-0.05 \pm 0.01$ \\
\hline & Inf & $-0.03 \pm 0.04$ & $0.05 \pm 0.02$ & $0.01 \pm 0.02$ \\
\hline & Sup & $0.06 \pm 0.05$ & $-0.04 \pm 0.05$ & $0.01 \pm 0.03$ \\
\hline & Mean & $-0.02 \pm 0.02$ & $-0.03 \pm 0.01$ & $-0.02 \pm 0.01$ \\
\hline
\end{tabular}


Table 7: The difference of Q value in $8 \mathrm{~mm}$ pre- and postoperatively.

\begin{tabular}{|c|c|c|c|c|c|c|c|}
\hline \multicolumn{3}{|c|}{ Items } & Nas & Tem & Inf & Sup & Mean \\
\hline \multirow[t]{16}{*}{ ZCB00 } & \multirow{4}{*}{$\begin{array}{c}\text { Preoperative Q \& } 1 \\
\text { week postoperative Q }\end{array}$} & Mean & $1.39 \pm 11.10$ & $0.02 \pm 0.11$ & $0.05 \pm 0.34$ & $0.02 \pm 0.41$ & $-0.00 \pm 0.23$ \\
\hline & & $\mathrm{r}$ & 0.056 & 0.693 & 0.225 & 0.335 & 0.328 \\
\hline & & $\mathrm{t}$ & 0.985 & 1.562 & 1.047 & 0.451 & -0.162 \\
\hline & & P value & 0.329 & 0.123 & 0.299 & 0.653 & 0.872 \\
\hline & \multirow{4}{*}{$\begin{array}{c}\text { Preoperative Q \&1 } \\
\text { month postoperative Q }\end{array}$} & Mean & $0.06 \pm 0.43$ & $0.04 \pm 0.13$ & $0.03 \pm 0.27$ & $-0.06 \pm 0.37$ & $0.02 \pm 0.15$ \\
\hline & & $\mathrm{r}$ & 0.491 & 0.770 & 0.410 & 0.298 & 0.632 \\
\hline & & $\mathrm{t}$ & 1.080 & 2.226 & 0.932 & -1.104 & 0.804 \\
\hline & & P value & 0.285 & 0.030 & 0.355 & 0.275 & 0.425 \\
\hline & \multirow{4}{*}{$\begin{array}{l}1 \text { week postoperative } \mathrm{Q} \\
\text { \&1 month postoperative } \\
\mathrm{Q}\end{array}$} & Mean & $-2.04 \pm 13.42$ & $-0.01 \pm 0.11$ & $-0.01 \pm 0.39$ & $-0.09 \pm 0.36$ & $0.02 \pm 0.26$ \\
\hline & & r & 0.250 & 0.622 & 0.203 & 0.309 & 0.161 \\
\hline & & $\mathrm{t}$ & -0.987 & -0.692 & -0.124 & -1.624 & 0.440 \\
\hline & & $P$ value & 0.330 & 0.493 & 0.902 & 0.112 & 0.663 \\
\hline & \multirow{4}{*}{$\begin{array}{c}1 \text { week postoperative } \\
\text { Q -Preoperative Q \& } 1 \\
\text { month postoperative Q } \\
\text {-Preoperative Q }\end{array}$} & Mean & $-2.04 \pm 13.42$ & $0.01 \pm 0.11$ & $-0.01 \pm 0.39$ & $-0.09 \pm 0.36$ & $0.02 \pm 0.26$ \\
\hline & & $\mathrm{r}$ & 0.135 & 0.504 & 0.326 & 0.664 & 0.318 \\
\hline & & $\mathrm{t}$ & -0.987 & 0.692 & -0.124 & -1.624 & 0.440 \\
\hline & & $P$ value & 0.330 & 0.493 & 0.902 & 0.112 & 0.663 \\
\hline \multirow[t]{16}{*}{ Zeiss 839} & \multirow{4}{*}{$\begin{array}{c}\text { Preoperative Q \& } 1 \\
\text { week postoperative Q }\end{array}$} & Mean & $-0.02 \pm 0.24$ & $0.04 \pm 0.13$ & $0.05 \pm 0.18$ & $0.03 \pm 0.38$ & $0.03 \pm 0.13$ \\
\hline & & r & 0.732 & 0.510 & 0.725 & 0.598 & 0.774 \\
\hline & & $\mathrm{t}$ & -0.658 & 2.414 & 2.197 & 0.713 & 2.030 \\
\hline & & $P$ value & 0.513 & 0.018 & 0.031 & 0.478 & 0.046 \\
\hline & \multirow{4}{*}{$\begin{array}{c}\text { Preoperative Q \&1 } \\
\text { month postoperative Q }\end{array}$} & Mean & $-0.01 \pm 0.31$ & $0.04 \pm 0.16$ & $-0.04 \pm 0.19$ & $0.10 \pm 0.32$ & $0.04 \pm 0.14$ \\
\hline & & $\mathrm{r}$ & 0.440 & 0.309 & 0.590 & 0.538 & 0.657 \\
\hline & & $\mathrm{t}$ & -0.183 & 2.027 & -1.567 & 2.476 & 1.888 \\
\hline & & $P$ value & 0.856 & 0.047 & 0.123 & 0.016 & 0.064 \\
\hline & \multirow{4}{*}{$\begin{array}{c}1 \text { week postoperative } \mathrm{Q} \\
\& 1 \text { month postoperative } \\
\mathrm{Q}\end{array}$} & Mean & $-0.00 \pm 0.26$ & $0.01 \pm 0.15$ & $-0.07 \pm 0.20$ & $0.02 \pm 0.43$ & $-0.01 \pm 0.17$ \\
\hline & & $r$ & 0.417 & 0.474 & 0.635 & 0.393 & 0.579 \\
\hline & & $\mathrm{t}$ & -0.011 & 0.625 & -2.478 & 0.283 & -0.560 \\
\hline & & $P$ value & 0.991 & 0.535 & 0.017 & 0.779 & 0.578 \\
\hline & \multirow{4}{*}{$\begin{array}{c}1 \text { week postoperative } \\
\text { Q -Preoperative Q \& } 1 \\
\text { month postoperative Q } \\
\text {-Preoperative Q }\end{array}$} & Mean & $0.04 \pm 0.12$ & $0.02 \pm 0.11$ & $-0.06 \pm 0.14$ & $0.00 \pm 0.42$ & $-0.00 \pm 0.11$ \\
\hline & & $r$ & 0.877 & 0.704 & 0.616 & 0.388 & 0.565 \\
\hline & & $\mathrm{t}$ & 2.239 & 1.084 & -3.036 & 0.071 & -0.118 \\
\hline & & $P$ value & 0.030 & 0.284 & 0.004 & 0.944 & 0.907 \\
\hline
\end{tabular}




\begin{tabular}{|c|c|c|c|c|c|c|c|}
\hline \multirow{16}{*}{$\begin{array}{l}\text { ZCB00 } \\
+ \text { Zeiss } \\
839\end{array}$} & \multirow{4}{*}{$\begin{array}{c}\text { Preoperative Q \& } 1 \\
\text { week postoperative Q }\end{array}$} & Mean & $0.63 \pm 7.55$ & $0.03 \pm 0.12$ & $0.05 \pm 0.27$ & $0.03 \pm 0.39$ & $0.01 \pm 0.19$ \\
\hline & & $\mathrm{r}$ & 0.061 & 0.600 & 0.485 & 0.516 & 0.571 \\
\hline & & $\mathrm{t}$ & 0.969 & 2.872 & 2.018 & 0.827 & 0.918 \\
\hline & & $P$ value & 0.334 & 0.005 & 0.046 & 0.410 & 0.360 \\
\hline & \multirow{4}{*}{$\begin{array}{c}\text { Preoperative Q \&1 } \\
\text { month postoperative Q }\end{array}$} & Mean & $0.03 \pm 0.34$ & $0.05 \pm 0.14$ & $-0.01 \pm 0.22$ & $-0.01 \pm 0.36$ & $0.02 \pm 0.13$ \\
\hline & & $\mathrm{r}$ & 0.565 & 0.640 & 0.554 & 0.491 & 0.744 \\
\hline & & $\mathrm{t}$ & 1.031 & 3.817 & -0.343 & -0.232 & 1.667 \\
\hline & & P value & 0.305 & 0.000 & 0.732 & 0.817 & 0.098 \\
\hline & \multirow{4}{*}{$\begin{array}{c}1 \text { week postoperative } Q \\
\& 1 \text { month postoperative } \\
Q\end{array}$} & Mean & $-0.95 \pm 9.27$ & $0.01 \pm 0.11$ & $-0.04 \pm 0.28$ & $-0.04 \pm 0.39$ & $0.01 \pm 0.19$ \\
\hline & & $r$ & 0.227 & 0.688 & 0.465 & 0.432 & 0.499 \\
\hline & & $\mathrm{t}$ & -0.966 & 1.267 & -1.170 & -0.975 & 0.352 \\
\hline & & $P$ value & 0.337 & 0.209 & 0.245 & 0.332 & 0.726 \\
\hline & \multirow{4}{*}{$\begin{array}{c}1 \text { week postoperative } \\
\text { Q -Preoperative } Q \text { \& } 1 \\
\text { month postoperative } Q \\
\text {-Preoperative } Q\end{array}$} & Mean & $-0.95 \pm 9.27$ & $0.01 \pm 0.11$ & $-0.04 \pm 0.28$ & $-0.04 \pm 0.39$ & $0.01 \pm 0.19$ \\
\hline & & $\mathrm{r}$ & 0.132 & 0.631 & 0.391 & 0.531 & 0.380 \\
\hline & & $\mathrm{t}$ & -0.966 & 1.267 & -1.170 & -0.975 & 0.352 \\
\hline & & $P$ value & 0.337 & 0.209 & 0.245 & 0.332 & 0.726 \\
\hline
\end{tabular}

Table 8: The $Q$ value in $9 \mathrm{~mm}$ in the two IOL groups (mean \pm SD).

\begin{tabular}{|c|c|c|c|c|}
\hline \multicolumn{2}{|l|}{ Items } & ZCB00 & Zeiss 839 & ZCB00+Zeiss 839 \\
\hline \multirow[t]{5}{*}{ Preoperative Q } & Nas & $-0.66 \pm 0.05$ & $-0.60 \pm 0.04$ & $-0.63 \pm 0.03$ \\
\hline & Tem & $-0.24 \pm 0.02$ & $-0.21 \pm 0.02$ & $-0.22 \pm 0.01$ \\
\hline & Inf & $-1.24 \pm 0.82$ & $-0.37 \pm 0.02$ & $-0.78 \pm 0.39$ \\
\hline & Sup & $-0.69 \pm 0.04$ & $-0.63 \pm 0.04$ & $-0.66 \pm 0.03$ \\
\hline & Mean & $-0.51 \pm 0.02$ & $-0.46 \pm 0.02$ & $-0.49 \pm 0.02$ \\
\hline \multirow[t]{5}{*}{1 week postoperative $Q$} & Nas & $-0.61 \pm 0.04$ & $-0.58 \pm 0.03$ & $-0.59 \pm 0.02$ \\
\hline & Tem & $-0.26 \pm 0.02$ & $-0.24 \pm 0.02$ & $-0.25 \pm 0.01$ \\
\hline & Inf & $-0.44 \pm 0.03$ & $-0.38 \pm 0.03$ & $-0.41 \pm 0.02$ \\
\hline & Sup & $-0.72 \pm 0.04$ & $-0.64 \pm 0.06$ & $-0.68 \pm 0.04$ \\
\hline & Mean & $-0.51 \pm 0.02$ & $-0.46 \pm 0.02$ & $-0.48 \pm 0.02$ \\
\hline \multirow[t]{5}{*}{1 month postoperative $Q$} & Nas & $-0.67 \pm 0.05$ & $-0.57 \pm 0.03$ & $-0.62 \pm 0.03$ \\
\hline & Tem & $-0.30 \pm 0.03$ & $-0.28 \pm 0.02$ & $-0.29 \pm 0.02$ \\
\hline & Inf & $-0.49 \pm 0.03$ & $-0.30 \pm 0.03$ & $-0.40 \pm 0.02$ \\
\hline & Sup & $-0.66 \pm 0.03$ & $-0.61 \pm 0.04$ & $-0.64 \pm 0.02$ \\
\hline & Mean & $-0.53 \pm 0.02$ & $-0.44 \pm 0.02$ & $-0.48 \pm 0.02$ \\
\hline \multirow{5}{*}{$\begin{array}{c}1 \text { week postoperative } Q \\
\text {-Preoperative } Q\end{array}$} & Nas & $0.06 \pm 0.06$ & $0.06 \pm 0.04$ & $0.05 \pm 0.03$ \\
\hline & Tem & $-0.02 \pm 0.02$ & $-0.06 \pm 0.02$ & $-0.03 \pm 0.01$ \\
\hline & Inf & $0.97 \pm 1.00$ & $-0.01 \pm 0.03$ & $0.44 \pm 0.46$ \\
\hline & Sup & $-0.01 \pm 0.05$ & $0.02 \pm 0.05$ & $-0.01 \pm 0.03$ \\
\hline & Mean & $-0.00 \pm 0.02$ & $0.00 \pm 0.02$ & $-0.00 \pm 0.01$ \\
\hline \multirow{5}{*}{$\begin{array}{c}1 \text { month postoperative } \mathrm{Q} \\
\text {-Preoperative } \mathrm{Q}\end{array}$} & Nas & $-0.02 \pm 0.05$ & $0.01 \pm 0.04$ & $-0.01 \pm 0.03$ \\
\hline & Tem & $-0.07 \pm 0.04$ & $-0.08 \pm 0.03$ & $-0.07 \pm 0.02$ \\
\hline & Inf & $1.06 \pm 1.12$ & $0.07 \pm 0.03$ & $0.55 \pm 0.55$ \\
\hline & Sup & $0.06 \pm 0.04$ & $0.03 \pm 0.06$ & $0.03 \pm 0.03$ \\
\hline & Mean & $-0.00 \pm 0.02$ & $0.01 \pm 0.02$ & $0.00 \pm 0.01$ \\
\hline
\end{tabular}


Table 9: The difference of Q value in $9 \mathrm{~mm}$ pre- and postoperatively.

\begin{tabular}{|c|c|c|c|c|c|c|c|}
\hline \multicolumn{3}{|c|}{ Items } & Nas & Tem & Inf & Sup & Mean \\
\hline \multirow[t]{16}{*}{ ZCB00 } & \multirow{4}{*}{$\begin{array}{c}\text { Preoperative } Q \text { \& } 1 \text { week } \\
\text { postoperative } Q\end{array}$} & Mean & $-0.06 \pm 0.50$ & $0.02 \pm 0.12$ & $-0.97 \pm 7.84$ & $0.01 \pm 0.38$ & $0.00 \pm 0.17$ \\
\hline & & $\mathrm{r}$ & 0.195 & 0.694 & -0.032 & 0.302 & 0.513 \\
\hline & & $\mathrm{t}$ & -0.899 & 0.971 & -0.972 & 0.238 & 0.008 \\
\hline & & $P$ value & 0.372 & 0.336 & 0.335 & 0.812 & 0.994 \\
\hline & \multirow{4}{*}{$\begin{array}{c}\text { Preoperative Q \&1 } \\
\text { month postoperative Q }\end{array}$} & Mean & $0.02 \pm 0.37$ & $0.07 \pm 0.30$ & $-1.06 \pm 8.31$ & $-0.06 \pm 0.31$ & $0.00 \pm 0.15$ \\
\hline & & $\mathrm{r}$ & 0.480 & 0.089 & -0.013 & 0.322 & 0.641 \\
\hline & & $\mathrm{t}$ & 0.403 & 1.818 & -0.948 & -1.426 & 0.223 \\
\hline & & $P$ value & 0.688 & 0.075 & 0.347 & 0.160 & 0.824 \\
\hline & \multirow{4}{*}{$\begin{array}{c}1 \text { week postoperative } Q \\
\& 1 \text { month postoperative } \\
Q\end{array}$} & Mean & $0.02 \pm 0.24$ & $0.02 \pm 0.12$ & $0.03 \pm 0.28$ & $-0.07 \pm 0.32$ & $-0.00 \pm 0.14$ \\
\hline & & $\mathrm{r}$ & 0.678 & 0.623 & 0.409 & 0.240 & 0.469 \\
\hline & & $\mathrm{t}$ & 0.615 & 1.173 & 0.736 & -1.399 & -0.011 \\
\hline & & $P$ value & 0.542 & 0.247 & 0.466 & 0.169 & 0.991 \\
\hline & \multirow{4}{*}{$\begin{array}{c}1 \text { week postoperative } \\
\text { Q -Preoperative Q \& } 1 \\
\text { month postoperative Q } \\
\text {-Preoperative Q }\end{array}$} & Mean & $0.02 \pm 0.24$ & $0.02 \pm 0.12$ & $0.03 \pm 0.28$ & $-0.07 \pm 0.32$ & $-0.00 \pm 0.14$ \\
\hline & & $r$ & 0.806 & 0.505 & 1.000 & 0.673 & 0.518 \\
\hline & & $\mathrm{t}$ & 0.615 & 1.173 & 0.736 & -1.399 & -0.011 \\
\hline & & $P$ value & 0.542 & 0.247 & 0.466 & 0.169 & 0.991 \\
\hline \multirow[t]{16}{*}{ Zeiss 839} & \multirow{4}{*}{$\begin{array}{c}\text { Preoperative Q \& } 1 \text { week } \\
\text { postoperative } \mathrm{Q}\end{array}$} & Mean & $-0.05 \pm 0.24$ & $0.04 \pm 0.14$ & $0.01 \pm 0.25$ & $0.00 \pm 0.43$ & $0.00 \pm 0.14$ \\
\hline & & $\mathrm{r}$ & 0.739 & 0.555 & 0.497 & 0.637 & 0.716 \\
\hline & & $\mathrm{t}$ & -1.659 & 2.386 & 0.463 & 0.086 & 0.257 \\
\hline & & $P$ value & 0.101 & 0.020 & 0.645 & 0.932 & 0.798 \\
\hline & \multirow{4}{*}{$\begin{array}{c}\text { Preoperative Q \&1 } \\
\text { month postoperative Q }\end{array}$} & Mean & $0.01 \pm 0.22$ & $0.07 \pm 0.17$ & $-0.05 \pm 0.20$ & $-0.01 \pm 0.36$ & $-0.01 \pm 0.13$ \\
\hline & & $\mathrm{r}$ & 0.726 & 0.422 & 0.576 & 0.507 & 0.775 \\
\hline & & $\mathrm{t}$ & 0.189 & 3.168 & -2.047 & -0.199 & -0.314 \\
\hline & & $P$ value & 0.851 & 0.002 & 0.045 & 0.843 & 0.755 \\
\hline & \multirow{4}{*}{$\begin{array}{c}1 \text { week postoperative } Q \\
\& 1 \text { month postoperative } \\
Q\end{array}$} & Mean & $0.05 \pm 0.15$ & $0.05 \pm 0.15$ & $-0.08 \pm 0.17$ & $-0.01 \pm 0.40$ & $-0.00 \pm 0.12$ \\
\hline & & $\mathrm{r}$ & 0.764 & 0.764 & 0.749 & 0.338 & 0.760 \\
\hline & & $\mathrm{t}$ & 2.273 & 2.273 & -3.243 & -0.106 & -0.208 \\
\hline & & P value & 0.028 & 0.028 & 0.002 & 0.916 & 0.836 \\
\hline & \multirow{4}{*}{$\begin{array}{c}1 \text { week postoperative } \\
\text { Q -Preoperative Q \& } 1 \\
\text { month postoperative Q } \\
\text {-Preoperative Q }\end{array}$} & Mean & $0.05 \pm 0.15$ & $0.02 \pm 0.12$ & $-0.08 \pm 0.17$ & $-0.01 \pm 0.40$ & $-0.00 \pm 0.12$ \\
\hline & & $\mathrm{r}$ & 0.801 & 0.755 & 0.705 & 0.393 & 0.412 \\
\hline & & $\mathrm{t}$ & 2.273 & 1.034 & -3.243 & -0.106 & -0.208 \\
\hline & & $P$ value & 0.028 & 0.307 & 0.002 & 0.916 & 0.836 \\
\hline
\end{tabular}




\begin{tabular}{|c|c|c|c|c|c|c|c|}
\hline \multirow{16}{*}{$\begin{array}{l}\text { ZCB00 } \\
+ \text { Zeiss } \\
839\end{array}$} & \multirow{4}{*}{$\begin{array}{c}\text { Preoperative } Q \text { \& } 1 \text { week } \\
\text { postoperative } Q\end{array}$} & Mean & $-0.05 \pm 0.38$ & $0.03 \pm 0.13$ & $-0.44 \pm 5.33$ & $0.01 \pm 0.40$ & $0.00 \pm 0.15$ \\
\hline & & $\mathrm{r}$ & 0.442 & 0.624 & 0.000 & 0.541 & 0.636 \\
\hline & & $\mathrm{t}$ & -1.565 & 2.462 & -0.955 & 0.218 & 0.179 \\
\hline & & $P$ value & 0.120 & 0.015 & 0.341 & 0.828 & 0.858 \\
\hline & \multirow{4}{*}{$\begin{array}{c}\text { Preoperative Q \&1 } \\
\text { month postoperative Q }\end{array}$} & Mean & $0.01 \pm 0.30$ & $0.07 \pm 0.24$ & $-0.55 \pm 5.82$ & $-0.03 \pm 0.33$ & $-0.00 \pm 0.14$ \\
\hline & & $\mathrm{r}$ & 0.576 & 0.204 & 0.041 & 0.451 & 0.722 \\
\hline & & $\mathrm{t}$ & 0.444 & 3.205 & -0.998 & -1.069 & -0.034 \\
\hline & & $P$ value & 0.658 & 0.002 & 0.321 & 0.287 & 0.973 \\
\hline & \multirow{4}{*}{$\begin{array}{c}1 \text { week postoperative } Q \\
\& 1 \text { month postoperative } \\
Q\end{array}$} & Mean & $0.04 \pm 0.20$ & $0.02 \pm 0.12$ & $-0.03 \pm 0.23$ & $-0.04 \pm 0.37$ & $-0.00 \pm 0.13$ \\
\hline & & $\mathrm{r}$ & 0.715 & 0.718 & 0.578 & 0.324 & 0.677 \\
\hline & & $\mathrm{t}$ & 1.779 & 1.570 & -1.079 & -0.928 & -0.149 \\
\hline & & $P$ value & 0.079 & 0.120 & 0.283 & 0.356 & 0.882 \\
\hline & \multirow{4}{*}{$\begin{array}{c}1 \text { week postoperative } \\
\text { Q -Preoperative Q \& } 1 \\
\text { month postoperative Q } \\
\text {-Preoperative Q }\end{array}$} & Mean & $0.04 \pm 0.20$ & $0.02 \pm 0.12$ & $-0.03 \pm 0.23$ & $-0.04 \pm 0.37$ & $-0.00 \pm 0.13$ \\
\hline & & $r$ & 0.803 & 0.678 & 0.999 & 0.520 & 0.474 \\
\hline & & $\mathrm{t}$ & 1.779 & 1.570 & -1.079 & -0.928 & -0.149 \\
\hline & & P value & 0.079 & 0.120 & 0.283 & 0.356 & 0.882 \\
\hline
\end{tabular}

Table 10: The $Q$ value in $10 \mathrm{~mm}$ in the two IOL groups (mean \pm SD).

\begin{tabular}{|c|c|c|c|c|}
\hline \multicolumn{2}{|l|}{ Items } & ZCB00 & Zeiss 839 & ZCB00+Zeiss 839 \\
\hline \multirow[t]{5}{*}{ Preoperative Q } & Nas & $-0.71 \pm 0.04$ & $-0.65 \pm 0.04$ & $-0.68 \pm 0.03$ \\
\hline & Tem & $-0.31 \pm 0.02$ & $-0.29 \pm 0.02$ & $-\mathbf{0 . 3 0} \pm 0.01$ \\
\hline & Inf & $-0.56 \pm 0.03$ & $-0.51 \pm 0.03$ & $-0.53 \pm 0.02$ \\
\hline & Sup & $-0.72 \pm 0.03$ & $-0.71 \pm 0.04$ & $-0.72 \pm 0.02$ \\
\hline & Mean & $-0.57 \pm 0.02$ & $-0.54 \pm 0.02$ & $-0.56 \pm 0.01$ \\
\hline \multirow[t]{5}{*}{1 week postoperative $Q$} & Nas & $-0.65 \pm 0.03$ & $-0.64 \pm 0.04$ & $-0.64 \pm 0.02$ \\
\hline & Tem & $-0.31 \pm 0.02$ & $-0.30 \pm 0.02$ & $-0.21 \pm 0.02$ \\
\hline & Inf & $-0.56 \pm 0.06$ & $-0.52 \pm 0.04$ & $-0.54 \pm 0.03$ \\
\hline & Sup & $-0.72 \pm 0.03$ & $-1.30 \pm 0.66$ & $-1.03 \pm 0.35$ \\
\hline & Mean & $-0.56 \pm 0.02$ & $-0.69 \pm 0.16$ & $-0.63 \pm 0.09$ \\
\hline \multirow[t]{5}{*}{1 month postoperative $Q$} & Nas & $-0.68 \pm 0.04$ & $-0.64 \pm 0.03$ & $-0.66 \pm 0.02$ \\
\hline & Tem & $-0.36 \pm 0.03$ & $-0.35 \pm 0.03$ & $-0.35 \pm 0.02$ \\
\hline & Inf & $-0.62 \pm 0.04$ & $-0.44 \pm 0.03$ & $-0.53 \pm 0.03$ \\
\hline & Sup & $-0.68 \pm 0.03$ & $-0.64 \pm 0.03$ & $-0.66 \pm 0.02$ \\
\hline & Mean & $-0.59 \pm 0.02$ & $-0.52 \pm 0.02$ & $-0.55 \pm 0.01$ \\
\hline \multirow{5}{*}{$\begin{array}{c}1 \text { week postoperative } Q \\
\text {-Preoperative } Q\end{array}$} & Nas & $0.05 \pm 0.05$ & $0.04 \pm 0.04$ & $0.05 \pm 0.03$ \\
\hline & Tem & $-0.02 \pm 0.02$ & $-0.02 \pm 0.02$ & $-0.02 \pm 0.01$ \\
\hline & Inf & $-0.01 \pm 0.06$ & $-0.01 \pm 0.04$ & $-0.01 \pm 0.04$ \\
\hline & Sup & $-0.01 \pm 0.05$ & $-0.58 \pm 0.65$ & $-0.32 \pm 0.35$ \\
\hline & Mean & $-0.00 \pm 0.03$ & $-0.14 \pm 0.17$ & $-0.08 \pm 0.09$ \\
\hline \multirow{5}{*}{$\begin{array}{c}1 \text { month postoperative } \mathrm{Q} \\
\text {-Preoperative } \mathrm{Q}\end{array}$} & Nas & $0.01 \pm 0.05$ & $0.01 \pm 0.03$ & $0.01 \pm 0.03$ \\
\hline & Tem & $-0.05 \pm 0.03$ & $-0.07 \pm 0.03$ & $-0.06 \pm 0.02$ \\
\hline & Inf & $-0.05 \pm 0.04$ & $0.05 \pm 0.03$ & $-0.00 \pm 0.03$ \\
\hline & Sup & $0.07 \pm 0.04$ & $0.04 \pm 0.04$ & $0.06 \pm 0.03$ \\
\hline & Mean & $-0.00 \pm 0.03$ & $0.01 \pm 0.02$ & $0.00 \pm 0.02$ \\
\hline
\end{tabular}


Table 11: The difference of $Q$ value in $10 \mathrm{~mm}$ pre- and postoperatively.

\begin{tabular}{|c|c|c|c|c|c|c|c|}
\hline \multicolumn{3}{|c|}{ Items } & Nas & Tem & Inf & Sup & Mean \\
\hline \multirow[t]{16}{*}{ ZCB00 } & \multirow{4}{*}{$\begin{array}{c}\text { Preoperative } \mathrm{Q} \& 1 \text { week } \\
\text { postoperative } \mathrm{Q}\end{array}$} & Mean & $-0.07 \pm 0.41$ & $0.01 \pm 0.15$ & $0.01 \pm 0.50$ & $0.00 \pm 0.37$ & $-0.01 \pm 0.21$ \\
\hline & & $r$ & 0.251 & 0.559 & 0.149 & 0.086 & 0.302 \\
\hline & & $\mathrm{t}$ & -1.270 & 0.636 & 0.102 & 0.021 & -0.272 \\
\hline & & $P$ value & 0.209 & 0.527 & 0.919 & 0.983 & 0.787 \\
\hline & \multirow{4}{*}{$\begin{array}{c}\text { Preoperative Q \&1 month } \\
\text { postoperative } \mathrm{Q}\end{array}$} & Mean & $-0.03 \pm 0.33$ & $0.05 \pm 0.19$ & $0.06 \pm 0.33$ & $-0.09 \pm 0.28$ & $-0.00 \pm 0.18$ \\
\hline & & $\mathrm{r}$ & 0.303 & 0.551 & 0.297 & 0.335 & 0.415 \\
\hline & & $\mathrm{t}$ & -0.683 & 1.844 & 1.284 & -2.391 & -0.100 \\
\hline & & $P$ value & 0.497 & 0.071 & 0.205 & 0.020 & 0.921 \\
\hline & \multirow{4}{*}{$\begin{array}{l}1 \text { week postoperative } Q \\
\& 1 \text { month postoperative } Q\end{array}$} & Mean & $0.01 \pm 0.31$ & $0.04 \pm 0.14$ & $0.03 \pm 0.37$ & $0.04 \pm 0.32$ & $-0.00 \pm 0.16$ \\
\hline & & $r$ & 0.295 & 0.549 & 0.720 & 0.146 & 0.443 \\
\hline & & $\mathrm{t}$ & 0.298 & 1.795 & 0.445 & 0.876 & -0.115 \\
\hline & & $P$ value & 0.767 & 0.080 & 0.659 & 0.386 & 0.909 \\
\hline & \multirow{4}{*}{$\begin{array}{c}1 \text { week postoperative } \\
\text { Q -Preoperative } Q \text { \& } 1 \\
\text { month postoperative } Q \\
\text {-Preoperative } Q\end{array}$} & Mean & $-0.04 \pm 0.34$ & $0.03 \pm 0.15$ & $0.02 \pm 0.37$ & $-0.05 \pm 0.32$ & $-0.01 \pm 0.18$ \\
\hline & & $r$ & 0.611 & 0.667 & 0.778 & 0.680 & 0.704 \\
\hline & & $\mathrm{t}$ & -0.726 & 1.352 & 0.344 & -1.035 & -0.313 \\
\hline & & $P$ value & 0.472 & 0.184 & 0.732 & 0.306 & 0.756 \\
\hline \multirow{16}{*}{$\begin{array}{c}\text { Zeiss } \\
839\end{array}$} & \multirow{4}{*}{$\begin{array}{c}\text { Preoperative Q \& } 1 \text { week } \\
\text { postoperative } Q\end{array}$} & Mean & $-0.04 \pm 0.37$ & $0.02 \pm 0.18$ & $0.01 \pm 0.38$ & $0.58 \pm 5.55$ & $0.14 \pm 1.41$ \\
\hline & & $\mathrm{r}$ & 0.382 & 0.505 & 0.149 & 0.157 & -0.141 \\
\hline & & $\mathrm{t}$ & -0.954 & 1.028 & 0.165 & 0.886 & 0.855 \\
\hline & & P value & 0.343 & 0.307 & 0.870 & 0.379 & 0.395 \\
\hline & \multirow{4}{*}{$\begin{array}{c}\text { Preoperative } Q \text { \& } 1 \text { month } \\
\text { postoperative } Q\end{array}$} & Mean & $0.03 \pm 0.33$ & $0.07 \pm 0.19$ & $-0.51 \pm 0.26$ & $-0.05 \pm 0.32$ & $-0.01 \pm 0.12$ \\
\hline & & $\mathrm{r}$ & 0.434 & 0.448 & 0.300 & 0.293 & 0.710 \\
\hline & & $\mathrm{t}$ & 0.593 & 2.866 & -1.480 & -1.102 & -0.444 \\
\hline & & $P$ value & 0.555 & 0.006 & 0.145 & 0.275 & 0.659 \\
\hline & \multirow{4}{*}{$\begin{array}{l}1 \text { week postoperative } Q \\
\text { \&1 month postoperative } Q\end{array}$} & Mean & $0.06 \pm 0.20$ & $0.02 \pm 0.15$ & $-0.08 \pm 0.27$ & $-0.01 \pm 0.32$ & $-0.00 \pm 0.14$ \\
\hline & & $\mathrm{r}$ & 0.597 & 0.724 & 0.367 & 0.244 & 0.647 \\
\hline & & $\mathrm{t}$ & 1.976 & 0.962 & -1.904 & -0.166 & -0.107 \\
\hline & & $P$ value & 0.054 & 0.341 & 0.063 & 0.869 & 0.915 \\
\hline & \multirow{4}{*}{$\begin{array}{c}1 \text { week postoperative } \\
\text { Q -Preoperative Q \& } 1 \\
\text { month postoperative } \mathrm{Q} \\
\text {-Preoperative Q }\end{array}$} & Mean & $0.06 \pm 0.20$ & $0.02 \pm 0.15$ & $-0.08 \pm 0.27$ & $-0.01 \pm 0.32$ & $-0.00 \pm 0.14$ \\
\hline & & $\mathrm{r}$ & 0.632 & 0.693 & 0.558 & 0.471 & 0.369 \\
\hline & & $\mathrm{t}$ & 1.976 & 0.962 & -1.904 & -0.166 & -0.107 \\
\hline & & P value & 0.054 & 0.341 & 0.063 & 0.869 & 0.915 \\
\hline
\end{tabular}




\begin{tabular}{|c|c|c|c|c|c|c|c|}
\hline \multirow{16}{*}{$\begin{array}{c}\text { ZCB00 } \\
+ \text { Zeiss } \\
839\end{array}$} & \multirow{4}{*}{$\begin{array}{c}\text { Preoperative Q \& } 1 \text { week } \\
\text { postoperative } \mathrm{Q}\end{array}$} & Mean & $-0.05 \pm 0.39$ & $0.02 \pm 0.17$ & $0.01 \pm 0.44$ & $0.31 \pm 4.09$ & $0.07 \pm 1.05$ \\
\hline & & $\mathrm{r}$ & 0.316 & 0.525 & 0.152 & 0.129 & -0.078 \\
\hline & & $\mathrm{t}$ & -1.580 & 1.207 & 0.185 & 0.886 & 0.811 \\
\hline & & $P$ value & 0.117 & 0.230 & 0.854 & 0.377 & 0.419 \\
\hline & \multirow{4}{*}{$\begin{array}{c}\text { Preoperative } \mathrm{Q} \& 1 \text { month } \\
\text { postoperative } \mathrm{Q}\end{array}$} & Mean & $-0.00 \pm 0.33$ & $0.06 \pm 0.19$ & $0.00 \pm 0.30$ & $-0.07 \pm 0.30$ & $-0.00 \pm 0.15$ \\
\hline & & r & 0.357 & 0.504 & 0.336 & 0.315 & 0.570 \\
\hline & & $\mathrm{t}$ & -0.058 & 3.344 & 0.061 & -2.390 & -0.332 \\
\hline & & $P$ value & 0.954 & 0.001 & 0.952 & 0.019 & 0.741 \\
\hline & \multirow{4}{*}{$\begin{array}{c}1 \text { week postoperative } \mathrm{Q} \\
\text { \&1 month postoperative } \mathrm{Q}\end{array}$} & Mean & $0.02 \pm 0.26$ & $0.03 \pm 0.14$ & $-0.03 \pm 0.33$ & $-0.02 \pm 0.32$ & $0.00 \pm 0.15$ \\
\hline & & $r$ & 0.425 & 0.667 & 0.625 & 0.221 & 0.576 \\
\hline & & $\mathrm{t}$ & 0.855 & 1.919 & -0.790 & -0.727 & 0.014 \\
\hline & & P value & 0.395 & 0.058 & 0.432 & 0.469 & 0.989 \\
\hline & \multirow{4}{*}{$\begin{array}{c}1 \text { week postoperative } \\
\text { Q -Preoperative Q \& } 1 \\
\text { month postoperative Q } \\
\text {-Preoperative Q }\end{array}$} & Mean & $0.01 \pm 0.28$ & $0.03 \pm 0.15$ & $-0.03 \pm 0.33$ & $-0.03 \pm 0.32$ & $-0.01 \pm 0.16$ \\
\hline & & $r$ & 0.599 & 0.686 & 0.688 & 0.585 & 0.626 \\
\hline & & $\mathrm{t}$ & 0.394 & 1.637 & -0.866 & -0.839 & -0.312 \\
\hline & & P value & 0.694 & 0.105 & 0.389 & 0.403 & 0.756 \\
\hline
\end{tabular}

Furthermore, the influence of different incision positions on corneal $Q$ value were explored (Table 12-14). At $6.0 \mathrm{~mm}$ corneal diameter, in ZCB00 group, there was no statistically significant difference among different incision positions in corneal $Q$ value at 1 week and 1 month postoperatively compared with the preoperative corneal Q value. In the Zeiss 839 group, surgical incision positions had a statistically significant influence on the mean corneal $\mathrm{Q}$ value $(\mathrm{P}=0.033)$ and the superior corneal $\mathrm{Q}$ value $(\mathrm{P}=0.045) 1$ week postoperatively. The incision positions were correlated with the mean corneal $Q$ value $(\beta=0.197, R 2=0.683, P=0.004)$ and the superior corneal $Q$ value $(\beta=0.260, R 2=0.476, P=0.004)$ at 1 week postoperatively. The Post-HOC analysis found that the superior incision had a significant effect on the reduction of the superior corneal $\mathrm{Q}$ value $(\mathrm{P}=0.015)$ and the mean corneal $\mathrm{Q}$ value $(\mathrm{P}=0.015) 1$ week postoperatively. In $\mathrm{ZCB} 00$ + Zeiss 839 group, surgical incision positions had statistical significance on the superior corneal $\mathrm{Qvalue}(\mathrm{P}=0.001)$ and the temporal corneal $\mathrm{Q}$ value $(\mathrm{P}=0.005) 1$ week postoperatively. The incision positions were correlated with the superior corneal $Q$ value $(\beta=-0.192, P=0.007)$ and the temporal corneal $\mathrm{Q}$ value $(\mathrm{R}=0.183, \mathrm{P}=0.034) 1$ week postoperatively. The Post-HOC analysis found that the temporal arc incision has a significant effect on the reduction of the temporal corneal $\mathrm{Q}$ value $(\mathrm{P}=0.003) 1$ week postoperatively, but the superior incision has no markedly effect on the reduction of the superior corneal $Q$ value.

At $7.0 \mathrm{~mm}$ corneal diameter, in $\mathrm{ZCB} 00$ group, there was no statistically significant difference among different incision positions in corneal Q value at 1 week and 1 month postoperatively compared with the preoperative corneal $Q$ value. In the Zeiss 839 group, surgical incision positions had a statistically significant influence on the mean corneal $(\mathrm{P}=0.029)$, the superior $(\mathrm{P}=0.025) 1$ week postoperatively and the temporal corneal $\mathrm{Q}$ value $(\mathrm{P}=0.049) 1$ month postoperatively. The incision positions were correlated with the mean $(\beta=0.208, P=0.002)$, the superior corneal $Q$ value $(\beta=0.260, P=0.004)$ at 1 week postoperatively and the temporal corneal $Q$ value $(\beta=0.213, P=0.085)$ at 1 month postoperatively. The Post-HOC analysis found that the superior incision had a significant effect on the reduction of the superior $(\mathrm{P}=0.013)$ and mean $(\mathrm{P}=0.016)$ corneal $\mathrm{Q}$ value 1 week postoperatively; and also had a markedly effect on the reduction of the temporal corneal $Q$ value at 1 month postoperatively $(\mathrm{P}=0.069)$. In $\mathrm{ZCB} 00+$ Zeiss 839 group, surgical incision positions had statistical significance on the superior corneal $\mathrm{Q}$ value $(\mathrm{P}=0.001) 1$ week postoperatively. The incision positions were correlated with the superior corneal $Q$ value $(\beta=-0.192, P=0.007)$ and the temporal corneal $Q$ value $(\beta=-0.204, P=0.005) 1$ week postoperatively. The Post-HOC analysis found that the superior incision has a significant effect on the reduction of the superior corneal Q value postoperatively.

The corneal Q value at diameter of $8-10 \mathrm{~mm}$, there was no statistically significant difference among different surgical incision positions in the IOL ZCB00 and IOL Zeiss 839 implanted eyes. 
Table 12: Influence of incision sites on temporal and mean Q value in $6 \mathrm{~mm}$.

\begin{tabular}{|c|c|c|c|c|c|c|c|}
\hline $\begin{array}{l}\text { IOL } \\
\text { types }\end{array}$ & Items & $\begin{array}{l}\text { superior } \\
\text { incision }\end{array}$ & $\begin{array}{c}\text { nasal } \\
\text { incision }\end{array}$ & $\begin{array}{c}\text { temporal } \\
\text { incision group }\end{array}$ & $\begin{array}{l}\text { nasal arc } \\
\text { incision } \\
\text { group }\end{array}$ & $\begin{array}{l}\text { temporal } \\
\text { arc incision } \\
\text { group }\end{array}$ & $\begin{array}{c}P \\
\text { value }\end{array}$ \\
\hline \multirow[t]{3}{*}{ ZCB00 } & $\begin{array}{c}\text { 1week postoperative } \mathrm{Q} \\
\text { (Mean) }\end{array}$ & $-0.29 \pm 0.16$ & $-0.21 \pm 0.04$ & $-0.42 \pm 0.09$ & $-0.24 \pm 0.19$ & $-0.31 \pm 0.24$ & 0.313 \\
\hline & $\begin{array}{c}\text { 1week postoperative } Q \\
(\text { Tem })\end{array}$ & $-0.11 \pm 0.13$ & $-0.11 \pm 0.05$ & $-0.24 \pm 0.18$ & $-0.09 \pm 0.12$ & $-0.06 \pm 0.13$ & 0.141 \\
\hline & $\begin{array}{c}\text { 1month postoperative } Q \\
\text { (Tem) }\end{array}$ & $-0.13 \pm 0.20$ & $-0.04 \pm 0.10$ & $-0.07 \pm 0.40$ & $-0.14 \pm 0.19$ & $-0.07 \pm 0.09$ & 0.422 \\
\hline \multirow[t]{14}{*}{$\begin{array}{c}\text { Zeiss } \\
839\end{array}$} & $\begin{array}{c}\text { 1week postoperative } Q \\
\text { (Mean) }\end{array}$ & $-0.35 \pm 0.25$ & $-0.14 \pm 0.16$ & $-0.21 \pm 0.23$ & $-0.19 \pm 0.17$ & $-0.19 \pm 0.23$ & 0.033 \\
\hline & $\begin{array}{c}\text { 1week postoperative } \\
\text { Q (Sup) }\end{array}$ & $-0.71 \pm 0.76$ & $-0.06 \pm 0.23$ & $-0.35 \pm 0.55$ & $-0.26 \pm 0.43$ & $-0.39 \pm 0.49$ & 0.045 \\
\hline & $\begin{array}{c}\text { 1month postoperative } Q \\
\text { (Mean) }\end{array}$ & $-0.23 \pm 0.12$ & $-0.12 \pm 0.12$ & $-0.25 \pm 0.21$ & $-0.23 \pm 0.16$ & $-0.20 \pm 0.20$ & 0.447 \\
\hline & $\begin{array}{c}\text { 1month postoperative } \mathrm{Q} \\
\text { (Sup) }\end{array}$ & $-0.41 \pm 0.24$ & $-0.21 \pm 0.26$ & $-0.42 \pm 0.54$ & $-0.37 \pm 0.41$ & $-0.50 \pm 0.37$ & 0.545 \\
\hline & $\begin{array}{l}\triangle 1 \text {-week postoperative } \\
\text { - preoperative } Q \text { value } \\
\text { (Nas) }\end{array}$ & $0.03 \pm 0.31$ & $-0.13 \pm 0.29$ & $0.05 \pm 0.18$ & $-0.10 \pm 0.20$ & $0.02 \pm 0.22$ & 0.183 \\
\hline & $\begin{array}{c}\triangle 1 \text {-month postoperative } \\
\text { - preoperative } Q \text { value } \\
\text { (Nas) }\end{array}$ & $-0.01 \pm 0.32$ & $0.01 \pm 0.21$ & $0.13 \pm 0.34$ & $-0.02 \pm 0.08$ & $0.06 \pm 0.20$ & 0.569 \\
\hline & $\begin{array}{c}\triangle 1 \text {-week postoperative } \\
\text { - preoperative } Q \text { value } \\
(\text { Tem })\end{array}$ & $0.02 \pm 0.09$ & $-0.01 \pm 0.07$ & $-0.08 \pm 0.13$ & $-0.05 \pm 0.16$ & $0.06 \pm 0.14$ & 0.023 \\
\hline & $\begin{array}{c}\triangle 1 \text {-month postoperative } \\
\text { - preoperative } Q \text { value } \\
\text { (Tem) }\end{array}$ & $0.03 \pm 0.09$ & $-0.02 \pm 0.12$ & $-0.09 \pm 0.15$ & $-0.01 \pm 0.10$ & $-0.03 \pm 0.14$ & 0.157 \\
\hline & $\begin{array}{l}\triangle 1 \text {-week postoperative - } \\
\text { preoperative } Q \text { value (Inf) }\end{array}$ & $-0.10 \pm 0.13$ & $-0.06 \pm 0.12$ & $0.08 \pm 0.46$ & $-0.06 \pm 0.16$ & $-0.03 \pm 0.14$ & 0.332 \\
\hline & $\begin{array}{l}\triangle 1 \text {-month postoperative - } \\
\text { preoperative } Q \text { value (Inf) }\end{array}$ & $0.00 \pm 0.15$ & $-0.01 \pm 0.11$ & $0.15 \pm 0.48$ & $-0.01 \pm 0.11$ & $-0.01 \pm 0.12$ & 0.574 \\
\hline & $\begin{array}{l}\triangle 1 \text {-week postoperative } \\
\text { - preoperative } Q \text { value } \\
\text { (Sup) }\end{array}$ & $-0.44 \pm 0.49$ & $-0.03 \pm 0.28$ & $-0.10 \pm 0.20$ & $-0.12 \pm 0.39$ & $0.09 \pm 0.46$ & 0.005 \\
\hline & $\begin{array}{c}\triangle 1 \text {-month postoperative } \\
\text { - preoperative } Q \text { value } \\
\text { (Sup) }\end{array}$ & $-0.25 \pm 0.12$ & $-0.13 \pm 0.24$ & $-0.07 \pm 0.36$ & $-0.16 \pm 0.33$ & $0.03 \pm 0.30$ & 0.177 \\
\hline & $\begin{array}{c}\triangle 1 \text {-week postoperative } \\
\text { - preoperative } Q \text { value } \\
\text { (Mean) }\end{array}$ & $-0.12 \pm 0.17$ & $-0.07 \pm 0.14$ & $-0.05 \pm 0.05$ & $-0.07 \pm 0.10$ & $0.03 \pm 0.14$ & 0.015 \\
\hline & $\begin{array}{c}\triangle 1 \text {-month postoperative } \\
\text { - preoperative } Q \text { value } \\
\text { (Mean) }\end{array}$ & $-0.06 \pm 0.09$ & $-0.05 \pm 0.08$ & $-0.02 \pm 0.14$ & $-0.05 \pm 0.08$ & $0.01 \pm 0.10$ & 0.514 \\
\hline
\end{tabular}




\begin{tabular}{|c|c|c|c|c|c|c|c|}
\hline \multirow{5}{*}{$\begin{array}{c}\text { ZCB00 } \\
+ \text { Zeiss } \\
839\end{array}$} & $\begin{array}{c}\text { 1week postoperative } \mathrm{Q} \\
\text { (Sup) }\end{array}$ & $1.37 \pm 0.17$ & $1.19 \pm 0.12$ & $1.31 \pm 0.21$ & $1.27 \pm 0.18$ & $1.30 \pm 0.16$ & 0.001 \\
\hline & $\begin{array}{c}\text { 1week postoperative } Q \\
\text { (Mean) }\end{array}$ & $-0.31 \pm 0.20$ & $-0.16 \pm 0.14$ & $-0.26 \pm 0.23$ & $-0.22 \pm 0.18$ & $-0.24 \pm 0.23$ & 0.147 \\
\hline & $\begin{array}{c}\text { 1week postoperative } Q \\
(\mathrm{Tem})\end{array}$ & $-0.11 \pm 0.02$ & $0.06 \pm 0.03$ & $-0.17 \pm 0.03$ & $-0.09 \pm 0.02$ & $-0.05 \pm 0.02$ & 0.005 \\
\hline & $\begin{array}{c}\text { In 1month postoperative } \\
\text { Q (Mean) }\end{array}$ & $0.35 \pm 0.12$ & $0.24 \pm 0.09$ & $0.31 \pm 0.15$ & $0.31 \pm 0.11$ & $0.29 \pm 0.15$ & 0.228 \\
\hline & $\begin{array}{c}\text { 1month postoperative } \mathrm{Q} \\
(\mathrm{Tem})\end{array}$ & $-0.11 \pm 0.17$ & $-0.03 \pm 0.07$ & $-0.19 \pm 0.18$ & $-0.09 \pm 0.15$ & $-0.06 \pm 0.17$ & 0.282 \\
\hline
\end{tabular}

Table13: Post-HOC analysis of $Q$ value in $6 \mathrm{~mm}$.

\begin{tabular}{|c|c|c|c|c|}
\hline IOL Type & Item & group & group & P \\
\hline \multirow{2}{*}{\begin{tabular}{c} 
Zeiss 839 \\
\cline { 2 - 5 }
\end{tabular}} & 1week postoperative Q (Mean) & Nasal & Temporal arc & 0.015 \\
\hline \multirow{2}{*}{$\begin{array}{c}\text { ZCB00+Zeiss } \\
839\end{array}$} & 1week postoperative Q (Sup) & Temporal arc & Superior & 0.015 \\
\cline { 2 - 5 } & 1week postoperative Q (Tem) & Temporal arc & Temporal & 0.003 \\
\cline { 2 - 5 } & 1week postoperative Q (Sup) & Superior & Nasal & 0.003 \\
\cline { 3 - 5 } & & & Nasal arc & 0.024 \\
\hline
\end{tabular}

Table14: Regression and relativity of $Q$ value in $6 \mathrm{~mm}$.

\begin{tabular}{|c|c|c|c|c|}
\hline \multirow{2}{*}{ IOL Type } & Item & $\boldsymbol{\beta}$ & $\mathbf{R}^{2}$ & $\mathbf{P}$ \\
\hline \multirow{7}{*}{ Zeiss 839 } & $\begin{array}{c}\text { Incision site with 1-week } \\
\text { postoperative Q value (mean) }\end{array}$ & 0.197 & 0.683 & 0.004 \\
\cline { 2 - 5 } & $\begin{array}{c}\text { Incision site with 1-week } \\
\text { postoperative Q value (Sup) }\end{array}$ & 0.260 & 0.476 & 0.004 \\
\cline { 2 - 5 } & $\begin{array}{c}\Delta 1 \text {-week postoperative - } \\
\text { preoperative Q value (Tem) }\end{array}$ & 0.054 & 0.003 & 0.647 \\
\cline { 2 - 5 } & $\begin{array}{c}\triangle 1 \text {-week postoperative - } \\
\text { preoperative Q value (Sup) }\end{array}$ & 0.364 & 0.132 & 0.001 \\
\cline { 2 - 5 } & $\begin{array}{c}\triangle 1 \text {-week postoperative - } \\
\text { preoperative Q value (Mean) }\end{array}$ & 0.350 & 0.122 & 0.002 \\
\hline \multirow{2}{*}{ ZCB00+Zeiss 839 } & $\begin{array}{c}\text { Incision site with 1-week } \\
\text { postoperative Q value (Tem) }\end{array}$ & - & $\mathrm{R}=0.183$ & 0.034 \\
\cline { 2 - 5 } & $\begin{array}{c}\text { Incision site with 1-week } \\
\text { postoperative Q value (Sup) }\end{array}$ & -0.192 & - & 0.007 \\
\hline
\end{tabular}


Table 15: Influence of incision sites on Q value in $7 \mathrm{~mm}$.

\begin{tabular}{|c|c|c|c|c|c|c|c|}
\hline $\begin{array}{l}\text { IOL } \\
\text { types }\end{array}$ & Items & $\begin{array}{l}\text { superior } \\
\text { incision }\end{array}$ & $\begin{array}{c}\text { nasal } \\
\text { incision }\end{array}$ & $\begin{array}{c}\text { temporal } \\
\text { incision group }\end{array}$ & $\begin{array}{c}\text { nasal arc } \\
\text { incision group }\end{array}$ & $\begin{array}{l}\text { temporal arc } \\
\text { incision group }\end{array}$ & $P$ value \\
\hline \multirow[t]{3}{*}{ ZCB00 } & $\begin{array}{c}1 \text { week } \\
\text { postoperative Q } \\
\text { (Tem) }\end{array}$ & $-0.14 \pm 0.16$ & $-0.13 \pm 0.06$ & $-0.27 \pm 0.14$ & $-0.15 \pm 0.12$ & $-0.08 \pm 0.10$ & 0.329 \\
\hline & $\begin{array}{c}\text { 1month } \\
\text { postoperative Q } \\
\text { (Tem) }\end{array}$ & $-0.17 \pm 0.16$ & $-0.07 \pm 0.06$ & $-0.07 \pm 0.23$ & $-0.21 \pm 0.23$ & $-0.14 \pm 0.13$ & 0.177 \\
\hline & $\begin{array}{c}1 \text { month } \\
\text { postoperative Q } \\
\text { (Sup) }\end{array}$ & $-0.53 \pm 0.28$ & $-0.38 \pm 0.36$ & $-0.54 \pm 0.56$ & $-0.48 \pm 0.27$ & $-0.50 \pm 0.42$ & 0.379 \\
\hline \multirow[t]{5}{*}{$\begin{array}{c}\text { Zeiss } \\
839\end{array}$} & $\begin{array}{c}\text { 1week } \\
\text { postoperative Q } \\
\text { (Mean) }\end{array}$ & $-0.39 \pm 0.19$ & $-0.22 \pm 0.17$ & $-0.31 \pm 0.23$ & $-0.24 \pm 0.18$ & $-0.26 \pm 0.21$ & 0.029 \\
\hline & $\begin{array}{c}\text { 1month } \\
\text { postoperative Q? } \\
\text { ?]?]? }\end{array}$ & $-0.31 \pm 0.12$ & $-0.19 \pm 0.12$ & $-0.32 \pm 0.23$ & $-0.28 \pm 0.13$ & $-0.27 \pm 0.18$ & 0.140 \\
\hline & $\begin{array}{c}\text { 1week } \\
\text { postoperative Q } \\
\text { (Sup) }\end{array}$ & $-0.69 \pm 0.46$ & $-0.23 \pm 0.22$ & $-0.50 \pm 0.58$ & $-0.32 \pm 0.46$ & $-0.48 \pm 0.44$ & 0.025 \\
\hline & $\begin{array}{c}\text { 1month } \\
\text { postoperative Q } \\
\text { (Sup) }\end{array}$ & $-0.49 \pm 0.24$ & $-0.37 \pm 0.29$ & $-0.51 \pm 0.50$ & $-0.41 \pm 0.31$ & $-0.59 \pm 0.33$ & 0.596 \\
\hline & $\begin{array}{c}\text { 1month } \\
\text { postoperative Q } \\
\text { (Tem) }\end{array}$ & $-0.12 \pm 0.10$ & $-0.06 \pm 0.04$ & $-0.26 \pm 0.13$ & $-0.12 \pm 0.10$ & $-0.14 \pm 0.19$ & 0.049 \\
\hline \multirow[t]{4}{*}{$\begin{array}{c}\text { ZCB00 } \\
+ \text { Zeiss } \\
839\end{array}$} & $\begin{array}{c}\text { 1week } \\
\text { postoperative Q } \\
\text { (Mean) }\end{array}$ & $-0.34 \pm 0.23$ & $-0.19 \pm 0.16$ & $-0.26 \pm 0.23$ & $-0.22 \pm 0.18$ & $-0.24 \pm 0.23$ & 0.273 \\
\hline & $\begin{array}{c}\text { 1month } \\
\text { postoperative Q } \\
\text { (Mean) }\end{array}$ & $-0.32 \pm 0.17$ & $-0.17 \pm 0.13$ & $-0.23 \pm 0.22$ & $-0.22 \pm 0.15$ & $-0.20 \pm 0.19$ & 0.123 \\
\hline & $\begin{array}{c}\text { 1week } \\
\text { postoperative Q } \\
\text { (Sup) }\end{array}$ & $-0.65 \pm 0.52$ & $-0.16 \pm 0.32$ & $-0.45 \pm 0.54$ & $-0.34 \pm 0.43$ & $-0.41 \pm 0.42$ & 0.001 \\
\hline & $\begin{array}{c}\text { 1month } \\
\text { postoperative Q } \\
\text { (Tem) }\end{array}$ & $-0.14 \pm 0.14$ & $-0.04 \pm 0.05$ & $-0.19 \pm 0.18$ & $-0.09 \pm 0.15$ & $-0.06 \pm 0.17$ & 0.212 \\
\hline
\end{tabular}


Table 16: Post-HOC analysis of Q value in $7 \mathrm{~mm}$.

\begin{tabular}{|l|c|c|c|c|}
\hline IOL Type & Items & Group & Group & P \\
\hline \multirow{2}{*}{ Zeiss 839 } & $\begin{array}{c}\text { Incision site with 1-week } \\
\text { postoperative Q value (mean) }\end{array}$ & Superior & Temporal arc & 0.013 \\
\cline { 2 - 5 } & $\begin{array}{c}\text { Incision site with 1-week } \\
\text { postoperative Q value (Sup) }\end{array}$ & Superior & Temporal arc & 0.016 \\
\cline { 2 - 5 } & $\begin{array}{c}\text { Incision site with 1-week } \\
\text { postoperative Q value (Tem) }\end{array}$ & Superior & Temporal & 0.069 \\
\hline ZCB00+Zeiss 839 & $\begin{array}{c}\text { Incision site with 1-week } \\
\text { postoperative Q value (Sup) }\end{array}$ & Superior & Nasal & 0.004 \\
\cline { 2 - 5 } & & & Nasal arc & 0.034 \\
\cline { 2 - 5 } & & & Temporal arc & 0.028 \\
\hline
\end{tabular}

Table 17: Regression and relativity of $Q$ value in $7 \mathrm{~mm}$.

\begin{tabular}{|c|c|c|c|c|}
\hline IOL tyoe & Items & $\beta$ & $\mathbf{R}^{2}$ & $\mathbf{P}$ \\
\hline \multirow[t]{5}{*}{ Zeiss 839} & Incision site with 1-week postoperative Q value (Sup) & 0.283 & - & 0.002 \\
\hline & $\begin{array}{l}\text { Incision site with 1-month postoperative } Q \text { value } \\
\qquad(\mathrm{Tem})\end{array}$ & - & $R=-0.064$ & 0.641 \\
\hline & $\begin{array}{l}\text { Incision site with 1-week postoperative } Q \text { value } \\
\text { (mean) }\end{array}$ & 0.208 & - & 0.002 \\
\hline & $\begin{array}{l}\text { Incision site with 1-month postoperative } Q \text { value } \\
\qquad \text { (Mean) }\end{array}$ & - & $R=-0.035$ & 0.796 \\
\hline & $\begin{array}{l}\text { Incision site with 1-month postoperative } \mathrm{Q} \text { value } \\
\qquad \text { (Tem) }\end{array}$ & 0.213 & - & 0.085 \\
\hline \multirow[t]{4}{*}{ ZCB00+Zeiss 839} & Incision site with 1-week postoperative Q value (Sup) & 0.204 & - & 0.005 \\
\hline & $\begin{array}{l}\text { Incision site with 1-week postoperative } \mathrm{Q} \text { value } \\
\text { (mean) }\end{array}$ & - & $\mathrm{R}=0.209$ & 0.015 \\
\hline & $\begin{array}{l}\text { Incision site with 1-week postoperative } Q \text { value } \\
\qquad(\mathrm{Tem})\end{array}$ & - & $\mathrm{R}=0.199$ & 0.02 \\
\hline & $\begin{array}{l}\text { Incision site with 1-month postoperative } \mathrm{Q} \text { value } \\
\qquad \text { (Mean) }\end{array}$ & - & $\mathrm{R}=0.218$ & 0.024 \\
\hline
\end{tabular}


Table 18: Influence of incision sites on $Q$ value in $8 \mathrm{~mm}$.

\begin{tabular}{|c|c|c|c|c|c|c|c|}
\hline $\begin{array}{l}\text { IOL } \\
\text { types }\end{array}$ & Items & $\begin{array}{l}\text { superior } \\
\text { incision }\end{array}$ & $\begin{array}{c}\text { nasal } \\
\text { incision }\end{array}$ & $\begin{array}{c}\text { temporal } \\
\text { incision group }\end{array}$ & $\begin{array}{c}\text { nasal arc } \\
\text { incision group }\end{array}$ & $\begin{array}{l}\text { temporal arc } \\
\text { incision group }\end{array}$ & $\begin{array}{c}P \\
\text { value }\end{array}$ \\
\hline ZCB00 & $\begin{array}{c}\text { 1month } \\
\text { postoperative Q } \\
(\mathrm{Tem})\end{array}$ & $-0.23 \pm 0.17$ & $-0.12 \pm 0.09$ & $-0.14 \pm 0.17$ & $-0.29 \pm 0.28$ & $-0.16 \pm 0.13$ & 0.472 \\
\hline \multirow[t]{5}{*}{$\begin{array}{l}\text { Zeiss } \\
839\end{array}$} & $\begin{array}{c}\text { 1week } \\
\text { postoperative Q } \\
(\mathrm{Tem})\end{array}$ & $-0.21 \pm 0.12$ & $-0.10 \pm 0.06$ & $-0.24 \pm 0.19$ & $-0.16 \pm 0.15$ & $-0.17 \pm 0.14$ & 0.063 \\
\hline & $\begin{array}{c}\text { 1week } \\
\text { postoperative Q } \\
\text { (Inf) }\end{array}$ & $-0.37 \pm 0.32$ & $-0.24 \pm 0.24$ & $-0.29 \pm 0.36$ & $-0.24 \pm 0.13$ & $-0.21 \pm 0.21$ & 0.502 \\
\hline & $\begin{array}{c}\text { 1week } \\
\text { postoperative Q } \\
\text { (Mean) }\end{array}$ & $-0.44 \pm 0.16$ & $-0.30 \pm 0.20$ & $-0.40 \pm 0.26$ & $-0.31 \pm 0.19$ & $-0.35 \pm 0.20$ & 0.068 \\
\hline & $\begin{array}{c}\text { 1month } \\
\text { postoperative Q } \\
(\mathrm{Tem})\end{array}$ & $-0.18 \pm 0.12$ & $-0.11 \pm 0.05$ & $-0.28 \pm 0.17$ & $-0.20 \pm 0.13$ & $-0.22 \pm 0.20$ & 0.277 \\
\hline & $\begin{array}{c}\text { 1month } \\
\text { postoperative Q } \\
\text { (Sup) }\end{array}$ & $-0.56 \pm 0.21$ & $-0.50 \pm 0.38$ & $-0.62 \pm 0.44$ & $-0.47 \pm 0.23$ & $-0.63 \pm 0.34$ & 0.790 \\
\hline \multirow[t]{3}{*}{$\begin{array}{c}\text { ZCB00 } \\
+ \text { Zeiss } \\
839\end{array}$} & $\begin{array}{c}\text { 1week } \\
\text { postoperative Q } \\
\text { (Tem) }\end{array}$ & $-0.20 \pm 0.14$ & $-0.12 \pm 0.08$ & $-0.25 \pm 0.18$ & $-0.17 \pm 0.12$ & $-0.16 \pm 0.14$ & 0.058 \\
\hline & $\begin{array}{c}\text { 1week } \\
\text { postoperative Q } \\
\text { (Inf) }\end{array}$ & $-0.32 \pm 0.27$ & $-0.22 \pm 0.24$ & $-0.29 \pm 0.31$ & $-0.27 \pm 0.23$ & $-0.32 \pm 0.39$ & 0.882 \\
\hline & $\begin{array}{c}\text { 1month } \\
\text { postoperative Q } \\
(\text { Tem) }\end{array}$ & $-0.21 \pm 0.16$ & $-0.11 \pm 0.06$ & $-0.25 \pm 0.18$ & $-0.24 \pm 0.22$ & $-0.20 \pm 0.17$ & 0.318 \\
\hline
\end{tabular}

Table 19: Influence of incision sites on $Q$ value in $9 \mathrm{~mm}$.

\begin{tabular}{|c|c|c|c|c|c|c|c|}
\hline $\begin{array}{c}\text { IOL } \\
\text { types }\end{array}$ & Items & $\begin{array}{l}\text { superior } \\
\text { incision }\end{array}$ & $\begin{array}{c}\text { nasal } \\
\text { incision }\end{array}$ & $\begin{array}{c}\text { temporal } \\
\text { incision group }\end{array}$ & $\begin{array}{c}\text { nasal arc } \\
\text { incision group }\end{array}$ & $\begin{array}{l}\text { temporal arc } \\
\text { incision group }\end{array}$ & $\begin{array}{c}P \\
\text { value }\end{array}$ \\
\hline \multirow[t]{3}{*}{$\begin{array}{c}\text { Zeiss } \\
839\end{array}$} & $\begin{array}{c}\text { 1week } \\
\text { postoperative Q } \\
\text { (Tem) }\end{array}$ & $-0.28 \pm 0.13$ & $-0.16 \pm 0.06$ & $-0.30 \pm 0.20$ & $-0.18 \pm 0.10$ & $-0.25 \pm 0.16$ & 0.063 \\
\hline & $\begin{array}{c}\text { 1month } \\
\text { postoperative Q } \\
\text { (Tem) }\end{array}$ & $-0.25 \pm 0.14$ & $-0.17 \pm 0.08$ & $-0.33 \pm 0.23$ & $-0.28 \pm 0.17$ & $-0.33 \pm 0.22$ & 0.258 \\
\hline & $\begin{array}{c}\text { 1month } \\
\text { postoperative Q } \\
\text { (Inf) }\end{array}$ & $-0.33 \pm 0.13$ & $-0.26 \pm 0.19$ & $-0.31 \pm 0.33$ & $-0.33 \pm 0.10$ & $-0.26 \pm 0.18$ & 0.526 \\
\hline \multirow[t]{2}{*}{$\begin{array}{l}\text { ZCB00 } \\
+ \text { Zeiss } \\
839\end{array}$} & $\begin{array}{c}\text { 1week } \\
\text { postoperative Q } \\
\text { (Tem) }\end{array}$ & $-0.26 \pm 0.16$ & $-0.17 \pm 0.07$ & $-0.31 \pm 0.18$ & $-0.23 \pm 0.16$ & $-0.24 \pm 0.17$ & 0.063 \\
\hline & $\begin{array}{c}\text { 1month } \\
\text { postoperative Q } \\
\text { (Tem) }\end{array}$ & $-0.29 \pm 0.18$ & $-0.17 \pm 0.08$ & $-0.31 \pm 0.21$ & $-0.33 \pm 0.25$ & $-0.29 \pm 0.20$ & 0.188 \\
\hline
\end{tabular}


Table 20: Influence of incision sites on $Q$ value in $10 \mathrm{~mm}$.

\begin{tabular}{|c|c|c|c|c|c|c|c|}
\hline $\begin{array}{l}\text { IOL } \\
\text { types }\end{array}$ & Items & $\begin{array}{l}\text { superior } \\
\text { incision }\end{array}$ & $\begin{array}{c}\text { nasal } \\
\text { incision }\end{array}$ & $\begin{array}{c}\text { temporal } \\
\text { incision group }\end{array}$ & $\begin{array}{c}\text { nasal arc } \\
\text { incision group }\end{array}$ & $\begin{array}{l}\text { temporal arc } \\
\text { incision group }\end{array}$ & $\begin{array}{c}P \\
\text { value }\end{array}$ \\
\hline ZCB00 & $\begin{array}{c}\text { 1month } \\
\text { postoperative Q } \\
\text { (Sup) }\end{array}$ & $-0.65 \pm 0.25$ & $-0.65 \pm 0.15$ & $-0.47 \pm 0.03$ & $-0.73 \pm 0.16$ & $-0.68 \pm 0.14$ & 0.740 \\
\hline $\begin{array}{c}\text { Zeiss } \\
839\end{array}$ & $\begin{array}{c}\text { 1month } \\
\text { postoperative Q } \\
\text { (Tem) }\end{array}$ & $-0.32 \pm 0.18$ & $-0.24 \pm 0.09$ & $-0.38 \pm 0.24$ & $-0.36 \pm 0.19$ & $-0.40 \pm 0.23$ & 0.527 \\
\hline \multirow[t]{2}{*}{$\begin{array}{l}\text { ZCB00 } \\
+ \text { Zeiss } \\
839\end{array}$} & $\begin{array}{c}\text { 1month } \\
\text { postoperative Q } \\
\text { (Sup) }\end{array}$ & $-0.66 \pm 0.23$ & $-0.62 \pm 0.24$ & $-0.58 \pm 0.30$ & $-0.68 \pm 0.14$ & $-0.67 \pm 0.21$ & 0.798 \\
\hline & $\begin{array}{c}\text { 1month } \\
\text { postoperative Q } \\
(\mathrm{Tem})\end{array}$ & $-0.36 \pm 0.17$ & $-0.22 \pm 0.07$ & $-0.35 \pm 0.22$ & $-0.40 \pm 0.24$ & $-0.37 \pm 0.22$ & 0.437 \\
\hline
\end{tabular}

\section{DISSCUSSION}

Form visiblized IOL implanted surgery postoperatively, the surgery effect gradually developed to glasses removal and clear vision with less aberrations, mostly the pursuit of good visual effect depends on the perfect design and application of multifocal and EDOF IOL. Until now, the cataract surgery has realized the refractive correction purpose with the further understanding of Angle kappa and corneal Q value. Review of the literatures, the corneal $Q$ value has a positive linear correlation with posterior corneal astigmatism, and the significant positive correlation was found between the primary spherical aberration [12]. And also, Jun Zhang et al, proved that $\mathrm{Q}$ value-guided femtosecond laser-assisted in situ keratomileusis introduced lower HOA and better stability than small-incision extraction [13]. As a result, corneal $Q$ value is coming to the forefront of interest of refractive-correction cataract surgeons.

In this study, we reviewed 158 eyes of patients who underwent cataract surgery: For the ZCB00 group, the preoperative mean $Q$ values of corneal anterior surface were $-0.23 \pm 0.19 \mathrm{~mm}$, $-0.32 \pm 0.02,-0.41 \pm 0.02,-0.51 \pm 0.02,-0.57 \pm 0.02$, respectively, when corneal diameters were $6 \mathrm{~mm}, 7 \mathrm{~mm}, 8 \mathrm{~mm}, 9 \mathrm{~mm}$, and $10 \mathrm{~mm}$, respectively. The mean corneal $\mathrm{Q}$ value decreased with the increase of corneal diameter, which was similar to the results obtained in previous literatures [14], which was also observed in the Zeiss 839 group.

According to the statistical results of nasal, temporal, inferior, superior and mean corneal Q values before and after surgery, it was found that when the corneal diameter was $6 \mathrm{~mm}$, the temporal corneal $\mathrm{Q}$ values and mean corneal $\mathrm{Q}$ values for ZCB00 decreased significantly 1 week after surgery compared with those before surgery, one month after the operation, the temporal Q value decreased significantly. The superior and the mean $\mathrm{Q}$ value were significantly decreased 1 week and 1 month after Zeiss 839 implantation, respectively. For all the affected eyes, the superior and the mean $Q$ values 1 week after the lens implantation were significantly lower than those before the surgery, and 1 month later, the temporal and the mean $Q$ values were significantly lower than those before the surgery.

When the corneal diameter was $7 \mathrm{~mm}$, the temporal $\mathrm{Q}$ value decreased significantly 1 week and 1 month after implantation, and the superior Q value decreased significantly 1 week after implantation. The $\mathrm{Q}$ values of the superior and mean side of ZEISS 839 group were significantly decreased 1 week after implantation, and the $Q$ values of the temporal side, upper side, and mean were significantly decreased 1 month after implantation, respectively. For all the patients, the $Q$ values of the upper side and the mean 1 week after surgery were significantly lower than those before surgery, and the $Q$ values of the temporal side and the mean 1 month after surgery were significantly lower than those before surgery.

The temporal Q value decreased significantly 1 month after surgery in ZCB00 at the $8 \mathrm{~mm}$ of corneal diameter. Temporal, inferior and mean $Q$ values were significantly decreased 1 week after Zeiss 839 IOL implantation, and temporal and inferior $Q$ values were significantly decreased 1 month after implantation. For all the affected eyes, the $Q$ values of the temporal side and the lower side decreased significantly 1 week after surgery, respectively, and the $Q$ value of the temporal side decreased significantly 1 month after surgery. 
When the corneal diameter was $9 \mathrm{~mm}$, the $\mathrm{Q}$ value of the temporal side was significantly decreased 1 week and 1 month after implantation, and the $Q$ value of the inferior side was significantly increased compared with that before operation. For all the affected eyes, the $Q$ value of temporal side 1 week and 1 month after surgery was significantly decreased compared with that before surgery.

As for the $10 \mathrm{~mm}$ of the cornea, the $\mathrm{Q}$ value of the superior increased 1 month after implantation of ZCB00. The temporal Q value decreased significantly 1 month after Zeiss 839 lens implantation. For all the affected eyes, the $Q$ values of temporal and superior decreased significantly 1 month after surgery compared with those before surgery.

From the above experimental results, we can see that the corneal $Q$ value in a certain direction changed after surgery, that is, the corneal morphology changed, and the superior corneal $\mathrm{Q}$ value and the mean corneal $\mathrm{Q}$ value decreased compared with the preoperative value, the cornea changed in a flat direction. In view of the above, we consider that the incision of cataract surgery causes changes in corneal morphology [15]; Hayashi et al. [16] studied the effect of clear cornea incision on corneal shape and found that the incision interrupts the collagen fibers which lower the corneal tension and the stress is redistributed, so that the obvious flatting could be found in anterior corneal surface. Compared changes in corneal shape in clear corneal incision with scleral incision in cataract surgery, although the changes in corneal shape and astigmatism occurred after corneal incision were greater than those in scleral incision, we could still find the wedge-shaped flattening of the whole anterior corneal and the steeping around the wound occurred at 2 days after surgery and persisted for 8 weeks in scleral group. In addition, the $Q$ value at 1 week after surgery is not statistically significant compared with the $Q$ value at 1 month after surgery, that is to say, the corneal morphology no longer shows significant changes and tends to be stable, which is consistent with the results of previous studies [15].

Further observation revealed that: the superior $Q$ value, the temporal $\mathrm{Q}$ value and the mean $\mathrm{Q}$ value among all corneal diameters changed significantly compared with preoperative values. A review of the literature showed that compared with incisions at other locations, the total ocular aberration was the smallest after surgery, and the total corneal aberration decreased significantly [17], Hayashi K et al. [18]compared changes in the shape of corneal between eyes with nasal and temporal clear incisions, the flattening in the total cornea was more prominent in the nasal group; this further confirms that the position of corneal incision may influence the $Q$ values through corneal shape changes: In our study, for ZCB00 and Zeiss 839 groups, the superior incisions and the temporal arc incisions of $6 \mathrm{~mm}$ and $7 \mathrm{~mm}$ had significant effects on the decrease of the superior corneal $Q$ value and the mean corneal $Q$ value after cataract surgery, while the incisions' effect on the postoperative corneal $Q$ value in corneal diameter of $8 \mathrm{~mm}$ to $10 \mathrm{~mm}$ was not significant. Which we propose that superior and temporal arc limbal incisions do obvious effect on the corneal shape, review of previous literature, $\mathrm{Q}$ value is linear correlated with spherical aberration, when corneal refractive index and the pupil diameter are stable, the corneal surface is flat, namely the cornea Q value is smaller, the postoperative corneal spherical aberration is reduced accordingly. However, there was no statistical correlation with total aberration, vertical comets, horizontal comets, etc [19]. Therefore, compared with incisions at other locations, the superior corneal incision is conducive to the improvement of postoperative visual quality.

\section{CONCLUSION}

To sum up, with the development of refractive cataract surgery technology, the pursuit of good vision from far to near and contrast sensitivity, and the reduction of abnormal phenomenon of light, the cornea $Q$ value reflecting parameters being we know, in the form of corneal Q-dependent design for operation helps to reduce the influence of the spherical aberration in human eye. Through the above study, we realized that the location of the incision affects the postoperative corneal $\mathrm{Q}$ value, and the superior incision is helpful to reduce the postoperative corneal $Q$ value so as to lower the corneal spherical aberration, the clear understanding of corneal $\mathrm{Q}$ value will better achieve the postoperative visual quality of cataract.

Declarations: None of the author has a conflict of interest to disclose.

\section{Funding: None}

\section{Number of tables: 20}

\section{Number of figures: 0}

Conflicts of Interest: The authors declare that the research was conducted in the absence of any commercial or financial relationships that could be construed as a potential conflict of interest.

Short title: Effect of limbal incision positions on corneal Q value. 
Data availability statement: The data used to support the findings of this study are available from the corresponding author upon request.

Statement: No animals were used in the research, please see the attachment for the human ethics document.

Author's contribution: Yuchen Wang and Zizhen Wang make substantial contributions to conception; Yuchen Wang and Zizhen Wang review the literatures, collect the data, and draft the manuscript; Ziyuan Liu, Yinan Liu and Wei Wang revise the review; Xiaoyong Chen gives final approval of the version to be submitted and any revised version.

Consent to publish: The participants have consented to the submission of the review to the journal.

\section{REFERENCE}

1. Zheng S, Ying J, Wang B, Xie Z, Huang X, et al. (2013). Three-dimensional model for human anterior corneal surface. Journal of biomedical optics. 18(6):65-72.

2. Douthwaite WA, Parkinson A. (2009). Precision of orbscan II assessment of anterior corneal curvature and asphericity. Journal of refractive surgery. 25(5):435-443.

3. Calossi A. (2007). Corneal asphericity and spherical aberration. Joural of refractive surgery. 23(5):505-514.

4. Gatinel D, Haouat M, Hoang-Xuan T. (2002). A review of mathematical descriptors of corneal asphericity. Journal francais d ophtalmolgie. 25(1):81-90.

5. Beiko GH. (2007). Personalized correction of spherical aberration in cataract surgery. Journal of cataract refractive surgery. 33(8):1455-1460.

6. He JC. (2014). Theoretical model of the contributions of corneal asphericity and anterior chamber depth to peripheral wavefront aberrations. Ophthalmic and physiological optics. 34(3):321-330.

7. Gonzalez-Meijome JM, Villa-Collar C, Montes-Mico R, Gomes A. (2007). Asphericity of the anterior human cornea with different corneal diameters. Journal of cataract refractive surgery. 33(3):465-473.

8. Whang WJ, Yoo YS, Kim HS, Yoon G. (2020). Influence of corneal spherical aberration on prediction error of the Haigis-L formula. Scientific reports. 10(1):6445.

9. Savini G, Hoffer KJ, Barboni P. (2015). Influence of corneal asphericity on the refractive outcome of intraocular lens implantation in cataract surgery. Journal of cataract refractive surgery. 41(4):785-789.
10. Nio YK, Jansonius NM, Fidler V, Geraghty E, Norrby S, et al. (2002). Spherical and irregular aberrations are important for the optimal performance of the human eye. Ophthalmic Physiological optics. 22(2):103-112.

11. Kanellopoulos AJ, Asimellis G. (2014). Clear-cornea cataract surgery: pupil size and shape changes, along with anterior chamber volume and depth changes. A Scheimpflug imaging study. Clinical ophthalmology. 8(2):2141-2150.

12. Li C, Zhang J, Yin X, Li J, Cao Y, et al. (2019). Distribution and related factors of corneal regularity and posterior corneal astigmatism in cataract patients. Clinical ophthalmology. 13(1):1341-1352.

13. Zhang J, Zheng L, Zhao X, Sun Y, Feng W, et al. (2019). Corneal aberrations after small-incision lenticule extraction versus $Q$ value-guided laser-assisted in situ keratomileusis. Medicine (Baltimore). 98(5):e14210.

14. Xiong Y, Li J, Wang N, Liu X, Wang Z, et al. (2017). The analysis of corneal asphericity ( $Q$ value) and its related factors of 1,683 Chinese eyes older than 30 years. PLoS One. 12(5):e0176913.

15. Wu D, Wang Y, Zhang L, Wei S, Tang X. (2014). Corneal biomechanical effects: small-incision lenticule extraction versus femtosecond laser-assisted laser in situ keratomileusis. Journal of cataract refractive surgery. 40(6):954-962.

16. Hayashi K, Yoshida M, Hayashi H. (2010). Corneal shape changes after $2.0-\mathrm{mm}$ or $3.0-\mathrm{mm}$ clear corneal versus scleral tunnel incision cataract surgery. Ophthalmology. 117(7):1313-1323.

17. Chu L, Zhao JY, Zhang JS, Meng J, Wang MW, et al. (2016). Optimal incision sites to reduce corneal aberration variations after small incision phacoemulsification cataract surgery. International journal of ophthalmology. $9(4): 540-545$.

18. Hayashi K, Sato T, Yoshida M, Yoshimura K. (2019). Corneal shape changes of the total and posterior cornea after temporal versus nasal clear corneal incision cataract surgery. British hournal of ophthalmology.103(2):181-185.

19. Li F, Zhao X, Niu Y, Wang W, Zhang Y. (2011). Correlation analysis of corneal Q-value and aberration in myopia population. Journal of Medical Forum. 32(6):30-32. 